\title{
A Thousand Earths: A Very Large Aperture, Ultralight Space Telescope Array for Atmospheric Biosignature Surveys
}

\author{
Dániel Apai ${ }^{1}\left(\mathbb{D}\right.$, Tom D. Milster ${ }^{2}$, Dae Wook Kim², Alex Bixel ${ }^{3}$ (D), Glenn Schneider ${ }^{3}$ (1D, Ronguang Liang $^{4}$, and \\ Jonathan Arenberg ${ }^{5}$ (i) \\ ${ }^{1}$ Steward Observatory and the Lunar and Planetary Laboratory, The University of Arizona, Tucson, AZ 85721, USA; apai@arizona.edu \\ 2 James C. Wyant College of Optical Sciences, The University of Arizona, Tucson, AZ 85721, USA \\ ${ }^{3}$ Steward Observatory, The University of Arizona, Tucson, AZ 85721, USA \\ ${ }^{4}$ College of Optical Sciences, The University of Arizona, Tucson, AZ 85721, USA \\ ${ }^{5}$ Northrop Grumman Aerospace Systems, Redondo Beach, CA 90278, USA \\ Received 2019 January 2; revised 2019 May 29; accepted 2019 May 31; published 2019 July 29
}

\begin{abstract}
An outstanding, multidisciplinary goal of modern science is the study of the diversity of potentially Earth-like planets and the search for life in them. This goal requires a bold new generation of space telescopes, but even the most ambitious designs yet hope to characterize several dozen potentially habitable planets. Such a sample may be too small to truly understand the complexity of exo-earths. We describe here a notional concept for a novel space observatory designed to characterize 1000 transiting exo-earth candidates. The Nautilus concept is based on an array of inflatable spacecraft carrying very large diameter $(8.5 \mathrm{~m})$, very low weight, multiorder diffractive optical elements (MODE lenses) as light-collecting elements. The mirrors typical to current space telescopes are replaced by MODE lenses with a 10 times lighter areal density that are 100 times less sensitive to misalignments, enabling lightweight structure. MODE lenses can be cost-effectively replicated through molding. The Nautilus mission concept has a potential to greatly reduce fabrication and launch costs and mission risks compared to the current space telescope paradigm through replicated components and identical, lightweight unit telescopes. Nautilus is designed to survey transiting exo-earths for biosignatures up to a distance of $300 \mathrm{pc}$, enabling a rigorous statistical exploration of the frequency and properties of life-bearing planets and the diversity of exo-earths.
\end{abstract}

Key words: astrobiology - instrumentation: miscellaneous - planets and satellites: atmospheres - planets and satellites: terrestrial planets - telescopes

\section{Introduction}

One of the pivotal questions of modern science is whether life is common in the universe. Answering this question will most likely require measuring the occurrence rate of habitable planets, understanding their diversity, sampling their atmospheres, and determining whether the observed atmospheric compositions can be explained without biological activity (see, e.g., Seager et al. 2016; Fujii et al. 2018; Kiang et al. 2018). Although the characterization of exo-earths is a key science goal of next-generation telescopes (Gaudi et al. 2018; The LUVOIR Team 2018; The OST Mission Concept Study Team 2018), due to the challenging nature of the observations, most proposed telescope concepts may not be able to accomplish this goal on target samples large enough to allow statistical exploration in a multidimensional parameter space (e.g., Ramirez et al. 2019). A challenge central to these observations is the intrinsic faintness of exoplanets, which is further complicated by the close angular proximity of their bright host stars (planet/star contrast).

One of the most fundamental properties of telescopes - and a limiting factor for many studies of faint extrasolar planets-is light-collecting area. For over a century-following the commissioning of the $1.02 \mathrm{~m}$ diameter Yerkes observatory refractor-every large telescope built used a primary mirror to collect light, but large mirrors $(D>2.5 \mathrm{~m})$ remained very difficult and expensive to fabricate, align, and operate. Figure 1 provides an overview of the evolution of light-collecting power and technology used over the past four centuries. While initially refracting and reflecting telescopes had been both utilized, reflectors proved to be scalable in size beyond refractors. Eventually, however, both refractors and reflectors reached the diameter beyond which their primary lightcollecting elements (lenses and mirrors) became too heavy to maintain their figures. While functional refractors never exceeded $1.1 \mathrm{~m}$ in diameter, functional monolithic mirrors could be built as large as $5 \mathrm{~m}$. Manufacturing large-diameter $(D)$ mirrors with very high optical quality capable of working at optical wavelengths $(\lambda)(D>6 \mathrm{~m}$ and $\lambda \simeq 0.5 \mu \mathrm{m})$ has been a technological challenge.

For ground-based telescopes, after a four-decade gap, the advent of segmented mirrors and ultralight (honeycomb) mirrors with computer-controlled surfaces enabled larger apertures. These technologies also enable the next generation of telescopes (Extremely Large Telescopes) with effective diameters between 24.5 and $39.3 \mathrm{~m}$ (Gilmozzi \& Spyromilio 2007; Johns et al. 2012; Sanders 2013). It is unclear whether the same technology could be utilized to build $100 \mathrm{~m}$ class ground-based telescopes.

In space, monolithic mirrors have been used for the largest visual/near-infrared astronomical telescopes, with the Hubble Space Telescope's (HST) $2.4 \mathrm{~m}$ diameter mirror being the largest such element. The James Webb Space Telescope (JWST; D $6.5 \mathrm{~m}$ ) and some future concepts (such as the Large UV/ Optical/IR Surveyor [LUVOIR]) envision building on this heritage but utilizing segmented and actively controlled mirrors.

While further slow, gradual increases in the diameter of segmented mirrors is possible, mirrors arguably remain the single most important bottleneck in astronomical telescopes. With the slow growth of aperture sizes, obvious next steps in astrophysics remain beyond reach: for example, the study of 


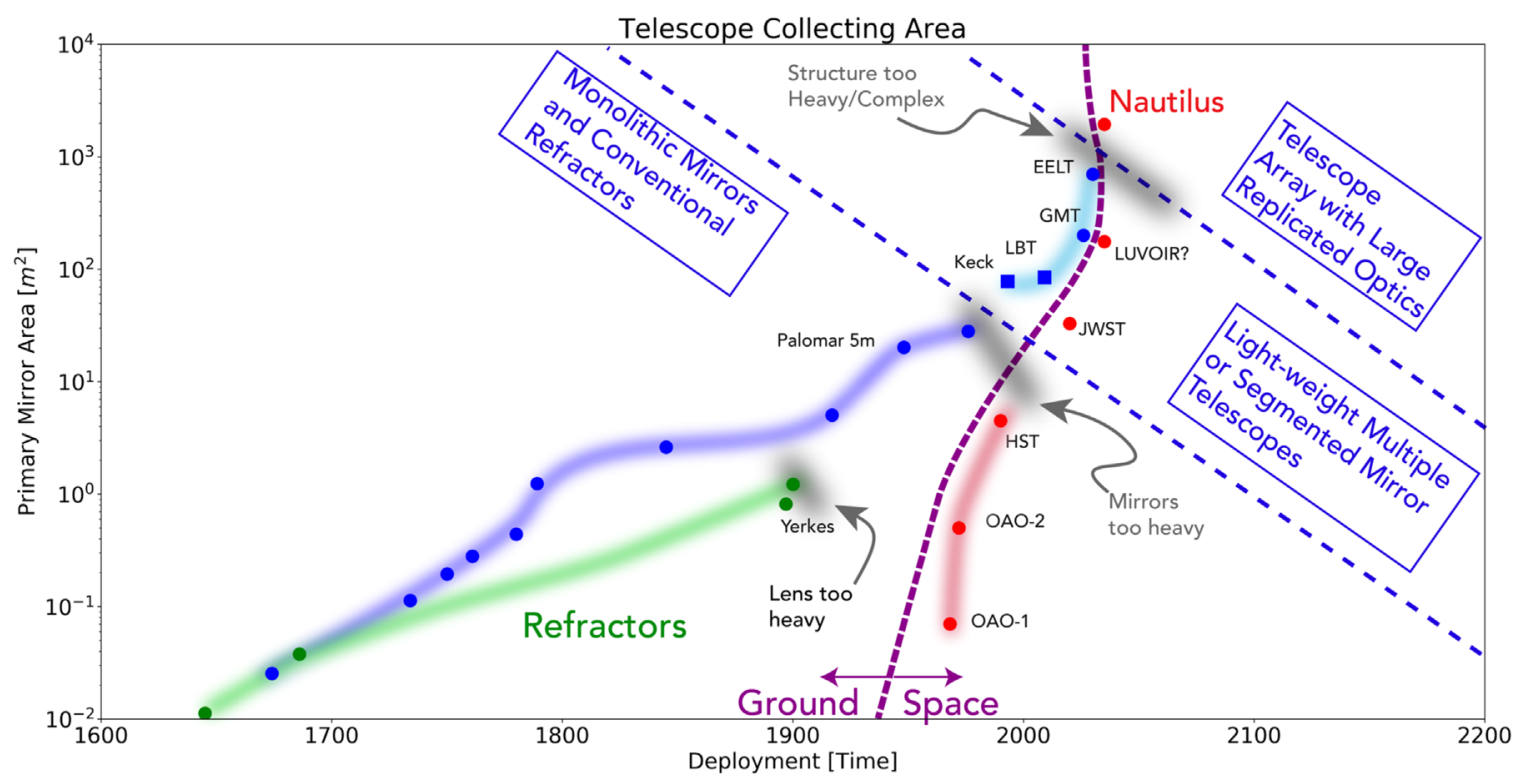

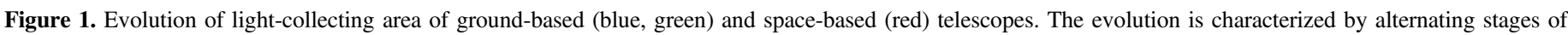

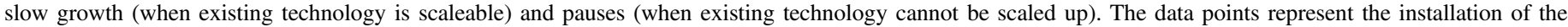

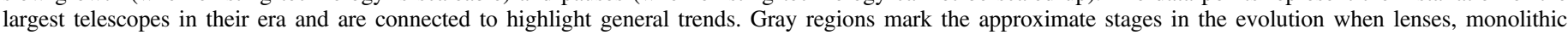

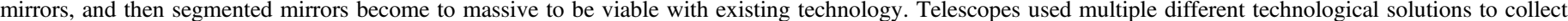
light. Large jumps in diameter are due to change in technology due to technological breakthroughs.

the diversity of Earth-like planets and assessing the frequency of atmospheric biosignatures in large samples $(N \sim 1000)$ of Earth-like planets remains beyond the reach of the telescopes envisioned even for the next 40 years.

We describe here a telescope concept that replaces the primary telescope mirror with multiorder diffractive engineered (MODE) material lens technology (Milster et al. 2018a, 2018b), offering a scalable solution for astronomical telescopes with low production, launch, and alignment costs compared to modern reflecting telescopes. Our mission concept is called Nautilus, named after J. Verne's submarine. Nautilus's science goal is to survey 1000 transiting, habitable zone, Earth-sized exoplanets to determine their atmospheric diversity and the occurrence rate of atmospheric biosignatures. This planet sample represents 1-2 orders of magnitude increase over the direct imaging and exoplanet transit telescope concepts currently envisioned for the next three decades (e.g., LUVOIR concept: The LUVOIR Team 2018; HabEx concept: Gaudi et al. 2018; OST concept: The OST Mission Concept Study Team 2018).

We first review the physical principles behind diffractive optical elements (Section 1.1), design considerations (Section 1.2), and the mass advantage (Section 1.3) they represent, and then we summarize relevant past telescopes and telescope concepts based on large diffractive optics (Section 1.4). In Section 2 we review the key elements of a large-scale atmospheric biosignature survey for Earth-like exoplanets, including the methodology, sample size, and definition (Sections 2.2 and 2.3), followed by simulated results (Section 2.4). This is followed by a summary of the science requirements (Section 3) for the survey and the baseline concept for the Nautilus telescope array (Section 3.1), including its launch, deployment, and operations. In Section 4.1 we discuss the fabrication and scalability of MODE lenses, current prototypes, and real-time optical quality assessment considerations for fabrication of large-scale diffractive lenses. Finally, we discuss how the Nautilus concept compares to the state of the art and discuss key advantages (reduced fabrication and launch costs, scalability) and the anticipated impact on astrophysics (Section 5).

\subsection{Principles of Large-scale Diffractive Optical Elements}

Diffractive optical elements perform lens-like functions, which can be analyzed with the principle of interference. For example, light transmitted through an aperture with radius $a$ that is illuminated by point source $P_{\text {src }}$ is conceptually illustrated in Figure 2(a). The aperture can be divided into equal-area Fresnel zones that identify which parts of the transmitted light interfere constructively at the observation point $P_{0}$ and which parts interfere destructively, as shown in Figure 2(b), based on the optical path difference (OPD) through point $Q$ of $\left(r_{\mathrm{src}}+r_{0}\right)-\left(z_{\mathrm{src}}+z_{0}\right)$. Boundaries of the Fresnel zones are defined by an increase of $\lambda / 2$ in OPD between successive zones. In this example, the first and second Fresnel zones produce a net zero light amplitude at the observation point, because light from an even-numbered zone combines destructively with light from an odd-numbered zone owing to the $\lambda / 2$ OPD between them. Likewise, light from the third and fourth zones combines destructively, leaving only light from the fifth zone to produce nonzero light amplitude at the observation point.

The well-known Fresnel zone plate (FZP) operates by blocking only the even or odd zones in the aperture, thus producing only constructive wave combination at the observation point. By extending this argument to off-axis illumination, it is understood that the FZP acts as a lens with a focus spot size that is equivalent to a classical lens of the same diameter and focal length. However, due to the fact that other focal positions can be identified along the axis, the classical FZP results in high-intensity background levels at the primary focus. In addition, since the constructive or destructive nature of the wave combination depends on wavelength, the focal point changes chromatically with a focal length proportional to $1 / \lambda$. That is, as wavelength increases, FZP focal length decreases, which is opposite the sense of a classical refractive lens 
(a)

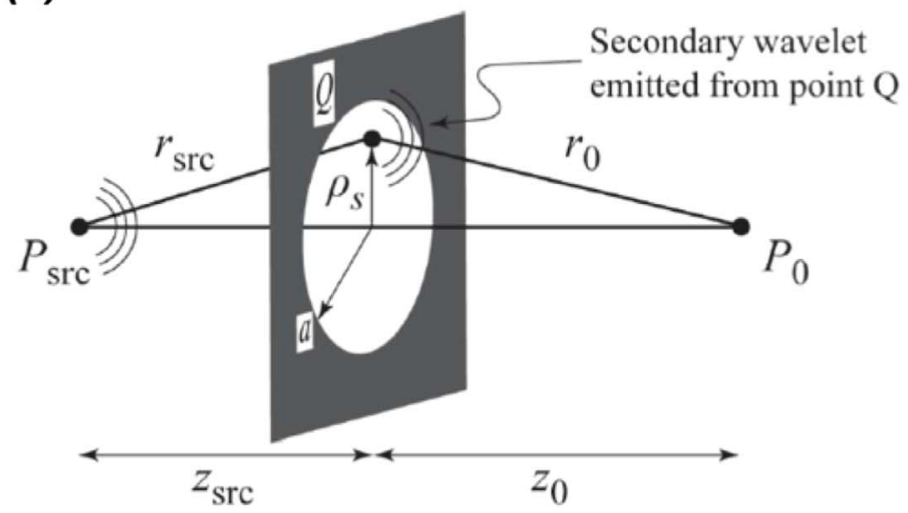

(b)

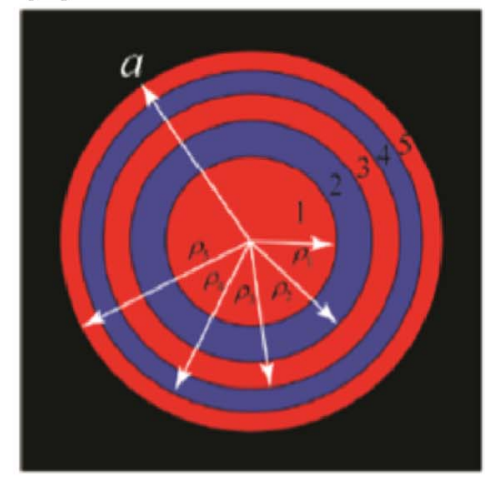

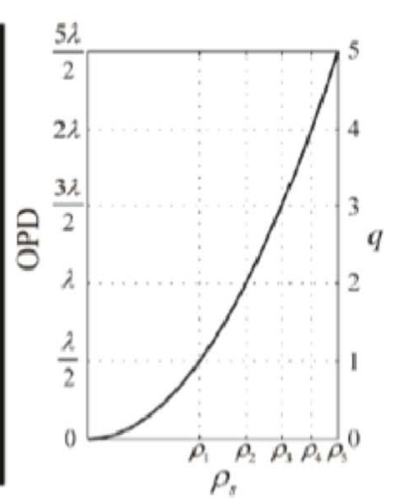

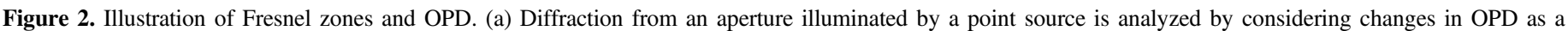
function of radius in the aperture. (b) Fresnel zone boundaries are defined by $\lambda / 2$ changes in OPD.

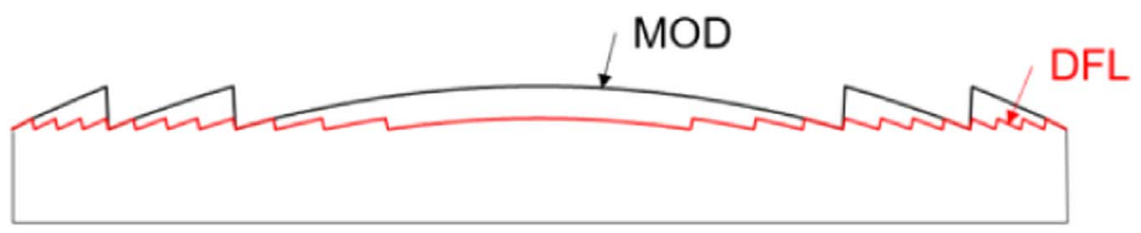

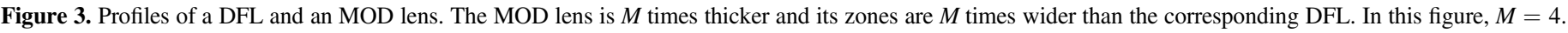

(Milster 2018). The combination of a properly designed FZP on a refractive singlet leads to compensating focal dispersions, which results in an achromatic singlet (Stone \& George 1988).

In order to increase the diffraction efficiency of light into the desired primary focal order, the FZP is replaced by a diffractive Fresnel lens (DFL), in which the opaque-zone FZP is replaced by a transmissive phase pattern that changes OPD as a function of radius. Neighboring zones are combined into a single quadratic phase surface, as shown in Figure 3. The profile in each zone pair has a maximum of one wavelength of OPD across it. Although the DFL has the same chromatic dispersion properties of a FZP, diffraction efficiency into the desired focal order is much greater. In fact, under ideal conditions, all of the light is focused into the primary order. Since the step height to achieve one wavelength of OPD at the transitions is very small (about $1 \mu \mathrm{m}$ for visible light), the DFL is an extremely thin, planar optical element.

In order to decrease chromatic focal dispersion, a multipleorder diffractive (MOD) lens was developed (Faklis \& Morris 1995). Instead of setting phase transitions based on a single wave of OPD, phase transitions are defined based on integer multiples of $M$ waves of OPD, where $M$ is the MOD number. As shown in Figure 3 for $M=4$, the MOD lens profile is thicker than the DFL by a factor of $M$ and zone spacings are increased. However, even if $M=1000$, the transition step is only about $1 \mathrm{~mm}$ high for a visible light design. The MOD lens operates over a set of higher diffracted orders where each order contains a wavelength of peak diffraction efficiency and each of these wavelengths comes to a common focus. The lens exhibits strong chromatic dispersion at intermediate wavelengths, but, interestingly, the maximum focal dispersion of the MOD lens is decreased to a range of approximately $f / M$ compared to the large range of a DFL. For example, an $f=5 \mathrm{~m}$ focal length $M=1000$ MOD would have a focal range of only $\pm 0.005 \mathrm{~m}$ over a wavelength range from
500 to $1000 \mathrm{~nm}$, where a DFL would have a focal range of approximately $\pm 3.0 \mathrm{~m}$ over the same range of wavelengths.

\subsection{Design Considerations for Multiorder Diffractive Engineered Lenses}

Design of a MODE lens for a particular application begins with the same desired first-order properties as a traditional refractive lens, such as the operating wavelengths and focal length. When designing for broadband performance, the design wavelength is the central wavelength of the wavelength range. Transition depths are defined based on the formula $M \frac{\lambda}{n_{2}-n_{1}}$, where $n_{2}$ and $n_{1}$ are the index of refraction of the lens material and the incident index, respectively. Transition locations are based on integer multiples of $M$ waves of OPD for on-axis rays. The individual zones are modeled and optimized in standard lens design software.

The change of refractive index with wavelength in each radial zone of the MOD surface also produces a chromatic focal shift, although it is much smaller in magnitude than for a DFL. This problem is compensated for by fabricating a weak DFL on the rear surface of the MOD lens to create an achromatic singlet for each MOD radial zone. This combination is what we call a MODE lens (Milster et al. 2018a, 2018b). The DFL is incorporated into the design by using a Sweatt model surface (Sweatt 1977) in lens design software, in which a fictitious glass with index approximately equal to the wavelength in $\mathrm{nm}$ is used to allow for significant optical power to exist in a very thin region. The Sweatt surface must later be converted to a physical surface by wrapping the OPD it produces. The MOD surface cannot be modeled as a Sweatt surface, as it no longer has a negligible physical thickness.

Following optimization in lens design software, the MODE design is verified using a physical optics simulation to confirm the diffractive performance. Optical path length is determined at the exit pupil reference sphere using ray tracing. A Hankel transform calculation is used to determine field values at a 


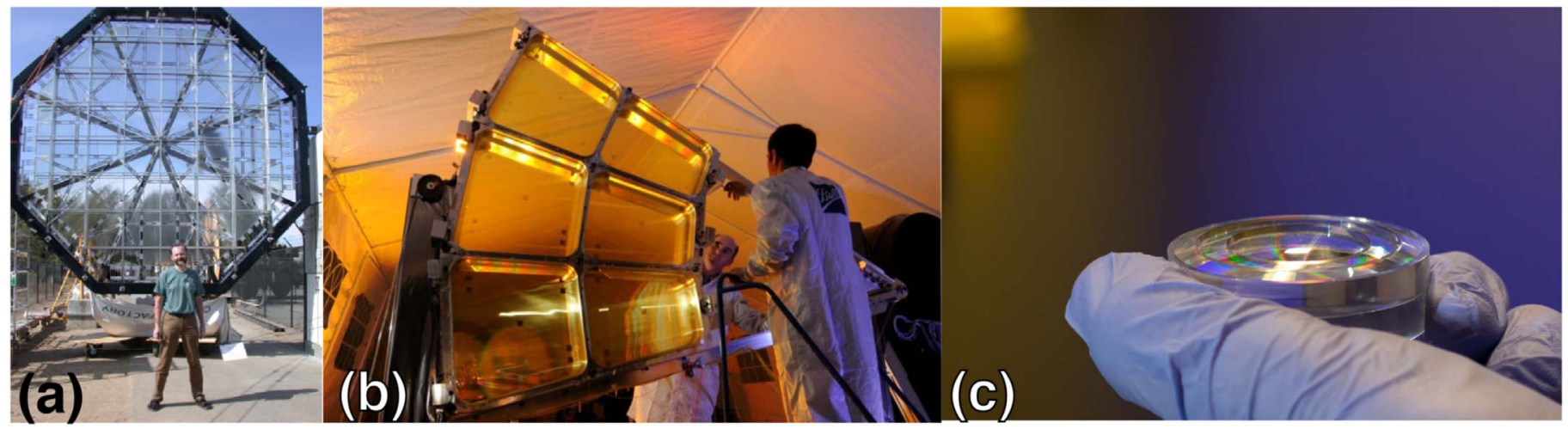

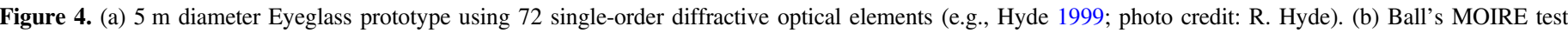

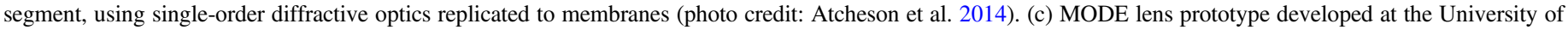
Arizona by our team.

sampled image plane. The magnitude squared of these field values provides the irradiance, which represents the pointspread function of the lens. This simulation is performed over a finely sampled spectrum of the full bandwidth, as well as for a range of image planes to account for both refractive and diffractive chromatic dispersion.

\subsection{Mass Advantages of MODE Lenses}

In this section we briefly explore the mass reduction achieved by MODE lenses compared to conventional lenses. The discussion presented here aims to provide a first, general approximation, from which individually designed MODE lenses may differ slightly.

We describe the mass of a conventional (refractive) lens as a planoconvex lens (sphere cap) as

$$
M_{\mathrm{lens}}=\rho \frac{1}{6} \pi h\left(3 R^{2}+h^{2}\right)
$$

where $\rho$ is the density of the glass, $h$ is the height of the lens, and $R$ is the radius of the lens. In contrast, the mass of a MODE lens (of order $M$ ) designed for a center wavelength $\lambda_{c}$ is simply

$$
M_{\text {mode }}=\phi \rho\left(R^{2} \pi\right)\left(M \lambda_{c}\right),
$$

where $\phi$ is the volume-filling factor of the MODE lens, close to 0.5 .

The mass ratio of a refractive lens to a MODE lens is then

$$
\frac{M_{\text {lens }}}{M_{\text {mode }}}=\frac{h\left(3 R^{2}+h^{2}\right)}{6 \phi R^{2} M \lambda_{c}} .
$$

For cases of relatively thin lenses $(h<R)$ this ratio can be approximated to the first order by

$$
\frac{M_{\text {lens }}}{M_{\text {mode }}} \approx \frac{h}{M \lambda_{c}} .
$$

For example, for a lens with a radius of $5 \mathrm{~m}$ and a relative thickness of $h / R=0.1$, an $M=1000$ MODE lens optimized for $\lambda_{c}=600 \mathrm{~nm}$ would provide about two orders of magnitude of mass reduction. The mass reduction by replacing a thick lens with a MODE lens would be even greater. In short, MODE lenses represent at least two orders of magnitude lower mass for a given lens diameter, a transformative advantage for space telescopes.

\subsection{Telescopes Based on Diffractive Optical Elements}

Diffractive optical elements (DOEs) are used for both space and commercial applications as small-scale internal optics. For example, the Lunar Orbiter Laser Altimeter mission uses a glass DOE as a beam splitter to divide a laser beam (Ramos-Izquierdo et al. 2009). The high-quality Canon EF telephoto zoom lenses incorporate two diffractive optical surfaces that are first diamond-turned in a mold and then replicated onto a curved glass substrate with epoxy resins (Nakai 2003). Similarly, Nikon's new Phase Fresnel (PF) high-end telephoto lens series utilizes a Fresnel lens in combination with a refractive group to allow large-aperture, high-quality, but very light photolenses.

The pioneering Eyeglass project is a very large aperture $(D=25-100 \mathrm{~m})$ diffractive space telescope concept developed by Lawrence Livermore National Laboratories (Hyde 1999; Hyde et al. 2002). Eyeglass uses a transmissive, DFL as its primary DOE light-collecting element. Corrective optics in a Schupmann configuration are used to provide broadband (470-700 nm) diffraction-limited imaging at visible wavelengths (Bernet \& Ritsch-Marte 2017). A $5 \mathrm{~m}$ diameter segmented (72 panels) prototype was built and successfully tested (see Figure 4), and extrapolations suggest 2-3 orders of magnitude lower weight per aperture area than that for HST's primary mirror $\left(180 \mathrm{~kg} \mathrm{~m}^{-2}\right.$ - in itself relatively low weight due to its "egg crate" structure). For Eyeglass options for the primary DOE material are thin sheets of glass or silica and films of polymers such as CPI or other fluorinated polyimides.

In a newer concept, the DARPA-funded Ball Aerospace project MOIRE (Membrane Optical Imager Real-Time Exploitation) aims to develop a $20 \mathrm{~m}$ aperture telescope using circular diffractive optics. MOIRE plans to deploy with a dedicated launch and capture live video and images of terrestrial targets in narrow spectral bandwidths $(\sim 30 \mathrm{~nm}$; see Hansen 2013$)$. The MOIRE project has been under development since 2010, and results from a $5 \mathrm{~m}$ scale brassboard instrument have recently been reported (Atcheson et al. 2014). The published plan is to make a glass master and then replicate membranes directly from it. The process used to make the MOIRE master is a multilevel lithographic approach.

Although Eyeglass and MOIRE demonstrate the interest and potential for very large aperture and relatively low cost space telescopes based on diffractive optics, neither of the designs is optimal for astrophysical applications, where broad wavelength coverage, faster optics (short relative focal lengths), and long operational lifetime are important. 


\section{A Large-scale Survey for Atmospheric Biosignatures}

The atmospheric characterization of extrasolar planets requires separating the light emitted or transmitted by the planet's atmosphere from that of its host star, which is-given the nearly 10 orders of magnitude visual brightness difference between the Earth and the Sun-a major observational challenge. Planet characterization methods separate planet light from its host's light spatially (e.g., Siegler 2018) or utilize temporal modulations. Spatial separation (extremely high contrast imaging) places extreme demands on the optical quality of the imaging system and will require highperformance coronagraphs (Trauger \& Traub 2007; Guyon et al. 2014) or an external occulter (e.g., Cash et al. 2005; Martin et al. 2018) to suppress starlight by a factor of $10^{10}$. The alternative approach, studies of temporal modulations, does not require very high optical quality but relies on high photometric precision. The rapid progress in semiconductor technology, combined with sophisticated instrument/telescope models, enabled very high precision photometry and spectroscopy from space-based telescopes. In fact, time-resolved differential photometry measurements have been the primary method through which Earth-sized planets have been discovered and characterized to date.

An important opportunity for the temporal separation of the planet light and starlight is offered in situations when the exoplanet passes in front of its host star. During such transit events starlight passes through and interacts with the planet's atmosphere. Differential measurements (comparing the spectra in and out of transit) can be used to constrain or determine the exoplanets' atmospheric composition (see, e.g., Seager \& Deming 2010).

A particularly important goal for exoplanet characterization is the search for atmospheric gases that may indicate the presence of life. Remote sensing of life is a notoriously difficult task: no single, unambiguous, easily detectable gas or signature has yet been identified (or expected to be found) that would reveal the presence of life (e.g., Seager et al. 2016; Kiang et al. 2018). Instead, combinations of inferred atmospheric absorbers may be interpreted-in the context of the planet's global properties (Apai et al. 2017) —as an indication for large-scale biological processes.

In this study we describe a general concept for a space telescope with atmospheric biosignature detection as its representative science goal and, given the exploratory nature of this study, will adopt a simplistic view on biosignature detection: we will focus on the ability to simultaneous detect several key absorbers in Earth analogs (e.g., $\mathrm{O}_{2}, \mathrm{O}_{3}$, and $\mathrm{H}_{2} \mathrm{O}$ in visible to short-wavelength near-infrared light). The detection of these species is not envisioned to be the final objective, but a measurement that is representative to the observational challenges and general trends characteristic to the problem. For more in-depth discussions of the different aspects of biosignatures we refer readers to comprehensive studies of biosignatures (Seager et al. 2016; Fujii et al. 2018; Meadows et al. 2018).

Although transit spectroscopy does not require high image quality, it can only be applied to planets with very low inclination orbits that transit their host stars as seen from Earth. Furthermore, transit spectroscopy requires large telescope apertures for two reasons: First, while the transit signal is a fixed fraction of the stellar flux, the signal-to-noise ratio $(\mathrm{S} / \mathrm{N})$ of the transit signal diminishes rapidly with the distance of the system from Earth. Second, in-transit data can only be collected during the relatively brief (typically $0.5-8 \mathrm{hr}$ long) planetary transit events, which occur approximately once a (terrestrial) year for Sun-like stars and more frequently for less massive (and thus intrinsically fainter) stars. Therefore, a large-scale transiting exoplanet survey will require both an efficient way to find transiting planets and a large-enough telescope that biosignatures for any given planet can be probed efficiently during only a small number of transit events. In the following we will review considerations for finding transiting exoplanets and for characterizing their atmospheric compositions.

\subsection{Science Goals of an Atmospheric Biosignature Survey}

Our baseline science goals are to simultaneously assess the diversity of possibly Earth-like planets and to carry out a statistically meaningful search for life on these worlds. The detection of biosignatures in the atmospheres of a sample of transiting Earth-sized planets (sample size $N_{\mathrm{pl}}$ ) is a necessary prerequisite to achieving these goals. In the following we will discuss the desired sample size, the wavelength range of interest, and the distance to which transits must be detected (as a function of sample size and composition) and explore the effective telescope diameter required to carry out the survey.

\subsection{Sample Size}

The sample size (number of planets studied, $N_{\mathrm{pl}}$ ) is a fundamental parameter for an exoplanet characterization and biosignature survey. The ideal sample size will depend on both the specific hypotheses to be tested and the degree of background knowledge completeness. Our current knowledge on the properties and composition of small (1-3 $\left.R_{\text {Earth }}\right)$ exoplanets is very limited (typically based only on measurements of stellar irradiation, mass, and bulk density; e.g., Rogers 2015; Fulton et al. 2017; Grimm et al. 2018). Such limited data and large uncertainties may only allow probabilistic assessment of the possible nature of the detected planets (e.g., Bixel \& Apai 2017; Catling et al. 2018), although this assessment can be supplemented by statistical predictions from planet formation models (Apai et al. 2018). Models predict that rocky planets with compositions different from Earth may be common and some classes may be habitable (e.g., waterworlds; Kite \& Ford 2018). Our current understanding of the diversity of exoplanet formation and evolution scenarios does not provide a robust basis for predicting the diversity of presentday exoplanet population.

Checlair et al. (2019) argue that, fundamentally, there are two approaches to exploring the diversity of exo-earths and to the search for life: one in which a direct extrapolation of solarsystem-type exoplanets are tested (i.e., search for Earth analogs), and one in which general hypotheses are tested. Checlair et al. (2019) show that the expected yield of LUVOIR could enable a statistical test of the predicted relationship between the carbon cycle efficiency and insolation, but our larger sample would allow us to observe this relationship over more dimensions such as planet size and stellar mass. A somewhat similar argument is laid down by Ramirez et al. (2019), who argue that understanding rocky planets as systems and identifying those that harbor life will likely require the study of hundreds if not thousands of planets: "We also suggest that next-generation missions are only the beginning of a much more data-filled era in the not-too-distant future, when possibly 
hundreds - thousands of HZ [habitable zone] planets will yield the statistical data we need to go beyond just finding habitable zone planets to actually determining which ones are most likely to exhibit life." Also consistent with this argument is the exoplanet community report by Apai et al. (2017), which highlights the need for large samples of exoplanets to be studied in order to build up the contextual knowledge necessary for understanding abiotic diversity and outliers of potentially biological nature.

The variety of processes and the range of key parameters involved in planet formation and subsequent evolution suggest that there is a strong likelihood for a great diversity in rocky planets-even without considering the possible impacts of extraterrestrial life on the evolution of the planetary atmosphere and climate. With this motivation we set the target sample size of potentially habitable planets to $N_{\mathrm{pl}}=1000$ for our science case. This sample size should be thought of as a sample size representative of the anticipated complexity of the parameter space exo-earths may occupy, rather than a well-determined value.

\subsection{Definition of the Planet Sample}

For transmission spectroscopy, the target selection fundamentally impacts the effective telescope collecting area (aperture) required, and it is thus a key property of any survey. In this section we explore several potential target samples and determine the distances at which the farthest target stars in each are located. The target selection consideration described below aims to demonstrate that viable options exist for multiple target samples, rather than to provide a final, optimized sample; additional considerations will lead to different target samples but should not affect the general feasibility.

In the following we will consider four different samples defined by host star spectral type distribution. For each of the samples we estimate the distance of the farthest host star, its brightness, and the relative and absolute amplitudes of the transit spectroscopy signal. In order to explore the range of target star distances, we first calculate the expected number of transiting Earth-sized planets as a function of stellar mass $\left(M_{*}\right.$, a proxy for stellar spectral type), distance (d) based on the volume density of stars $\left(\rho\left(M_{*}\right)\right)$, the occurrence rate of Earthsized habitable zone planets $\left(\eta_{\oplus}\left(M_{*}\right)\right)$, and the probability of these planets transiting $\left(P_{\mathrm{tr}}\right)$ :

$$
N\left(d, M_{*}\right)=\frac{4}{3} \pi d^{3} \times \rho\left(M_{*}\right) \times \eta_{\oplus}\left(M_{*}\right) \times P_{\mathrm{tr}}
$$

The transit probability is $P_{\mathrm{tr}}=R_{*} / a_{\mathrm{HZ}}$, where $a_{\mathrm{HZ}}$ is the semimajor axis of the habitable zone. The volume density of stars of different spectral types is calculated based on the RECONS sample of the local $10 \mathrm{pc}$ volume (Henry et al. 2018). We follow the model of Kopparapu et al. (2013) for the habitable zone boundaries, adopt their optimistic boundaries, and assume that the planets have transit probabilities that are the mean of the transit probabilities of planets at the inner and outer boundaries of the habitable zone. With a self-developed program-utilizing the astropy (Astropy Collaboration et al. 2013, 2018) and numpy (van der Walt et al. 2011) librarieswe calculated the properties of stars and planets in potential target samples, including distance, transit probability, transit depths, apparent brightness, and relative and absolute transit signals. Table 7 captures the key assumptions of our calculations.
In Figure 5 we show the cumulative number of transiting, Earth-sized, habitable zone planets as a function of distance and host spectral type (dotted curves). We also show four possible target samples selected from these planets. Sample 1 (red dotted curve) consists of the closest 1000 transiting Earthsized habitable zone planets. This sample is dominated by planets orbiting M-type host stars (blue dotted curve), but the stars in this sample are confined to within $\sim 55 \mathrm{pc}$ within the solar system. Our Sample 2 consists of up to 500 M-dwarf planets, supplemented by planets around FGK hosts. Stars in this sample are within $\sim 130 \mathrm{pc}$ of the solar system. Our Sample 3 consists of only planets of FGK-type stars (no M-dwarf planets). In this sample, to reach 1000 planets, we need to include stars up to $160 \mathrm{pc}$. In our Sample 4 we included only planets orbiting broadly Sun-like stars (G spectral type). With this criteria our targets are located up to $330 \mathrm{pc}$ away (see top panel of Figure 5). Table 1 summarizes the spectral type distribution in each sample, as well as the distance of the farthest star and the brightness of the faintest star in each sample. The table also provides the period range of the habitable zone planets for the target sample.

Exoplanet transit observations are relative measurements: the signal strength measured is relative to the apparent brightness of the host star and the ratio of the planet's and the star's projected surface areas. As the four samples defined in our study contain stars of very different sizes, brightness, and typical distances, the comparison of the samples in terms of the ease of detectability is nontrivial. The bottom panel of Figure 5 shows the absolute flux density difference during the transit of an Earth-sized planet around the target stars, as a function of spectral type and distance. We note that the flux density difference shown on the $y$-axis is continuum transit depth and not specific spectral features (which are typically several orders of magnitude fainter). For each of the curves (for stars of different spectral types) we also mark the faintest (most distant) stars in the target samples (as determined and marked in the top panel of the same figure). The dashed lines parallel with the $x$-axis denote the flux density levels corresponding to the transits around the faintest stars in the sample.

Interestingly, the out-of-transit to in-transit difference signal (in flux density) predicted for the faintest stars in all four samples falls within a relatively narrow range: $(2-5) \times 10^{-9} \mathrm{erg} \mathrm{cm}^{-2} \mathrm{~s}^{-1}$. This finding suggests that the absolute differential photometric precision required to detect a single transit of an Earth-sized planet around the faintest host stars in each sample is very similar between the samples, and that the precision floor is primarily set by the target sample size. However, because the number of transits of habitable zone planets that occur within a given time window and the brightness of the host stars (and therefore the required precision) are strongly spectral type dependent, the four samples discussed above are not equally well suited for a survey. We will consider these factors further in Section 2.4.

Considering the connections between stellar luminosity, habitable planet occurrence rate, transit probability, transit depth, and the volume (number) density of stars of different spectral types, we evaluated target definition choices and the resulting absolute signal strength. Our three key conclusions from the target selection study are as follows: (1) In the case of a single transit the resulting absolute signal strengthcorresponding to the transit of an Earth-sized planet-is insensitive to the spectral type distribution of the target stars. (2) For a survey limited by single-transit absolute signal 

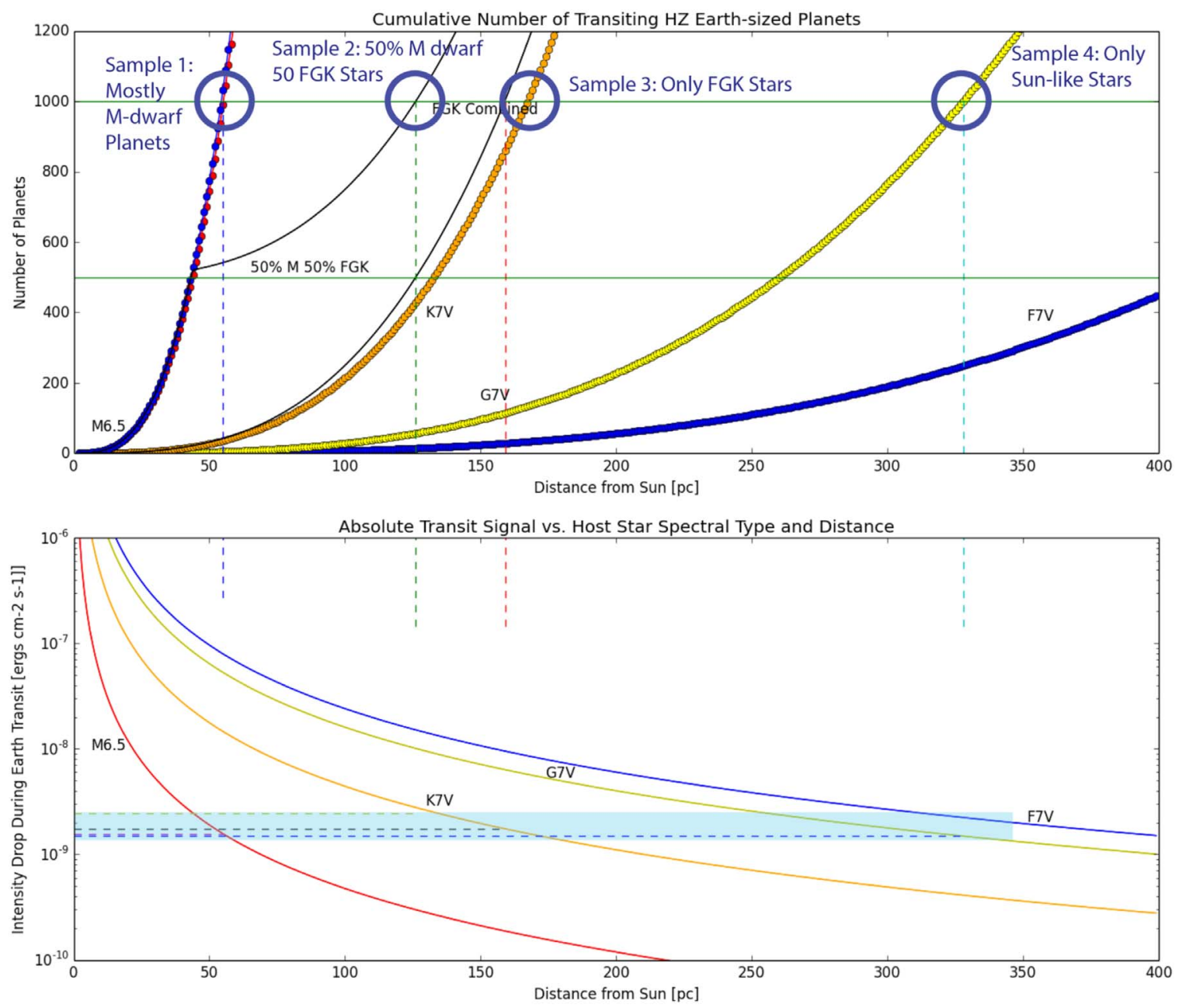

Figure 5. Top panel: cumulative number of transiting, Earth-sized planets around F stars (blue curve), G stars (yellow curve), K stars (orange curve), and M-type host stars (red curve) as a function of distance from the Sun. Four possible samples of 1000 transiting Earth-sized planets are shown: Sample 1 includes the closest 1000 transiting Earth-sized planets and will be dominated by planets orbiting M-dwarf host stars. Sample 2 will include up to 500 M-dwarf planets. Sample 3 includes only planets orbiting FGK-type stars. Sample 4 only includes broadly Sun-like stars. The maximum distances of stars in the four samples are 55, 130, 160, and 330 pc. Bottom panel: absolute signal (intensity drop during transit) as a function of host star distance and spectral type (for transits of Earth-sized planets). The vertical dashed lines show the distance of the most distant star in each sample. The shaded region shows the level of the faintest absolute signal in the four samples (transits around the faintest host stars).

Table 1

Properties of Target Samples

\begin{tabular}{|c|c|c|c|c|c|c|c|}
\hline \multirow{2}{*}{$\begin{array}{l}\text { Sample } \\
\text { ID }\end{array}$} & \multicolumn{4}{|c|}{ Planets around Stars } & \multirow{2}{*}{$\begin{array}{l}\text { Max. } \\
\text { Dist. } \\
\text { (pc) }\end{array}$} & \multirow{2}{*}{$\begin{array}{c}\text { Faintest } \\
I \text {-mag }\end{array}$} & \multirow{2}{*}{$\begin{array}{l}\text { HZ Period } \\
\text { (day) }\end{array}$} \\
\hline & M & K & G & F & & & \\
\hline 1 & 1000 & 0 & 0 & 0 & 55 & 16.5 & $4-18$ \\
\hline 2 & 500 & 438 & 58 & 14 & 126 & 15.9 & $4-1370$ \\
\hline 3 & 0 & 876 & 116 & 29 & 159 & 12.6 & $60-1370$ \\
\hline 4 & 0 & 0 & 1000 & 0 & 328 & 12.0 & $200-740$ \\
\hline
\end{tabular}

Note. The target samples include very close to 1000 habitable zone Earth-sized planets but reflect different choices in the exoplanet host stars.

strength, the key parameter of the survey definition is the sample size. (3) Considering the order-of-magnitude shorter orbital periods of habitable zone planets around M-type host stars and the deeper (relative) transit depths, for a survey limited by total telescope time and by relative photometric precision, a sample rich in M-type host stars may be advantageous. Such a sample is also optimal if the transiting planets must be located by the survey before the spectroscopic characterization can begin (due to the shorter transit periods around $\mathrm{M} \mathrm{dwarfs,} \mathrm{much} \mathrm{shorter} \mathrm{temporal} \mathrm{coverage} \mathrm{is} \mathrm{required}$ to detect or exclude transits). We note, however, that more detailed future studies are warranted to assess the impact of other factors not considered here, including stellar activity and the photometric variability it causes, and low-level stellar contamination of the transit spectra due to stellar heterogeneities (Rackham et al. 2018), which affect Sun-like stars much less than M dwarfs (Rackham et al. 2019).

\subsection{Transmission Spectrum Simulations}

We utilize the Planetary Spectrum Generator ${ }^{6}$ (PSG; Villanueva et al. 2018) to provide an approximate assessment of the expected quality of the spectra obtained by the telescope concept and explore what equivalent telescope diameter is required for the Nautilus observatory to ensure the detectability of $\mathrm{O}_{2}, \mathrm{O}_{3}, \mathrm{H}_{2} \mathrm{O}$, and $\mathrm{CO}_{2}$ absorption features in our target sample. The PSG is capable of simulating observations of model atmospheres from a variety of viewing geometries,

\footnotetext{
6 https://psg.gsfc.nasa.gov
} 
Table 2

Parameters of the Four Models Run through the Planetary Spectrum Generator

\begin{tabular}{lccccccc}
\hline \hline Host Type & $\begin{array}{c}M_{*} \\
\left(M_{\odot}\right)\end{array}$ & $\begin{array}{c}R_{*} \\
\left(R_{\odot}\right)\end{array}$ & $\begin{array}{c}T_{\text {eff }} \\
(\mathrm{K})\end{array}$ & $\begin{array}{c}\text { Distance } \\
(\mathrm{pc})\end{array}$ & $\begin{array}{c}\text { Semimajor Axis } \\
(\mathrm{au})\end{array}$ & $\begin{array}{c}\text { Transit Duration } \\
(\text { minutes })\end{array}$ & $\begin{array}{c}\text { No. of Observations } \\
(\mathrm{nm})\end{array}$ \\
\hline $\mathrm{M}$ & 0.20 & 0.28 & 3020 & 10 & 0.12 & 170 & 10 \\
$\mathrm{M}$ & 0.20 & 0.28 & 3020 & 50 & 0.12 & 170 & 10 \\
$\mathrm{G}$ & 1.00 & 1.00 & 5780 & 100 & 1.3 & 900 & 5 \\
$\mathrm{G}$ & 1.00 & 1.00 & 5780 & 300 & 1.3 & 900 & 5 \\
\hline
\end{tabular}

Note. In all cases we use the default profile of an Earth-like atmosphere with $R_{\mathrm{pl}}=1 R_{\oplus}$. The uncertainties shown are calculated for a $35 \times 8.5 \mathrm{~m}$ array of telescopes with continuous $1 \mathrm{~s}$ exposures over the duration of the transit, and we inflate the uncertainties by $20 \%$ to account for limb-darkening degeneracy. Finally, we reduce the uncertainties to account for the benefit of multiple transit observations.

either for planets within the solar system or around distant host stars of varying spectral types.

We run the PSG for the four models presented in Table 2. These include a mid-M dwarf and solar analog, each at a nearby and far distance based on the relative abundance of transiting planets in Figure 5 (10 and $50 \mathrm{pc}$ for the $\mathrm{M}$ dwarf, 100 and $300 \mathrm{pc}$ for the $\mathrm{G}$ dwarf; see Table 2). In all models, the planet is an Earth-sized, Earth-mass planet with the PSG's default profile for the Earth's atmosphere. The orbit is placed in the middle of the habitable zone as estimated from the host star's properties by Kopparapu et al. (2014). The viewing geometry is set to "Observatory" mode, with a planetary inclination of $90^{\circ}$ and orbital phase of $180^{\circ}$, and the viewing distance set to one of the four values in Table 2 .

The PSG can incorporate both Poisson and instrumental (e.g., readout) sources of noise, but we ignore the instrumental noise to focus on the effect of aperture size. The Poisson noise is calculated for a telescope array, each taking $1 \mathrm{~s}$ exposures. We set the number of exposures such that the total exposure time equals the transit duration, and generate a spectrum from 200 to $1800 \mathrm{~nm}$ with $1 \mathrm{~nm}$ resolution for each of the four models in Table 2.

The output of the PSG for the viewing geometry described above is the fraction of light blocked by the planet as a function of wavelength, with uncertainty estimates in each bin. The uncertainty estimates correspond to the amount of light collected over the entire transit duration. We take this to be the uncertainty on the transit depth, but we inflate the uncertainties by $20 \%$ to account for limb-darkening degeneracy. We assume that each planet will be observed in transit multiple times-10 times over the course of a year for the M-dwarf planets, and 5 times over the course of $\sim 7 \mathrm{yr}$ for the $\mathrm{G}$ dwarf planets-and we reduce the uncertainties by $\sqrt{N_{\mathrm{obs}}}$ for $N_{\text {obs }}$ observations. The justification for the number of revisits for each type of host star is explored in Section 2.5. We varied the telescope array configuration to explore the equivalent collecting area that satisfies our science goal.

Finally, in each spectral bin we draw a value from the normal distribution defined by the uncertainty to simulate the transmission spectrum achieved for each of the four models. The simulated spectra are presented in Figure 6 and binned for visibility according to Table 2. We find that with a 35-element array of 8.5 m diameter telescopes three partial biosignatures- $-\mathrm{O}_{3}, \mathrm{H}_{2} \mathrm{O}$, and potentially $\mathrm{O}_{2}$ - could be identified in the atmosphere of an Earth twin orbiting a nearby solar-type star. For nearby M-type hosts, all of these plus $\mathrm{CO}_{2}$ could be identified with high confidence. Furthermore, detection of $\mathrm{O}_{3}$ and $\mathrm{H}_{2} \mathrm{O}$ could be achieved for the most distant planets in the sample, allowing for a statistical analysis of the presence of biosignatures for hundreds of planets. Although our simulations show a broader wavelength range, we conclude that a narrower range (such as $500-1000 \mathrm{~nm}$ ) is sufficient for simultaneously detecting three key atmospheric components $\left(\mathrm{O}_{3}, \mathrm{H}_{2} \mathrm{O}, \mathrm{O}_{2}\right)$ of an Earth analog.

\subsection{Number of Visits}

Transit spectroscopy benefits from repeated transit observations. Combining the transmission spectra from several transits decreases uncertainties by $\sim \sqrt{N_{\text {obs }}}$. This strategy has been employed several times from the ground (e.g., Rackham et al. 2018; Bixel et al. 2019) and space (e.g., Kreidberg et al. 2014; de Wit et al. 2018; Zhang et al. 2018). A similar approach could be followed to achieve low- to moderate-S/N spectra of the atmospheres of Earth-sized planets in systems such as TRAPPIST-1 with JWST (Batalha et al. 2018).

When possible, it is therefore optimal to observe a planet's spectrum during every transit. A candidate exo-earth in the middle of the habitable zone of a Sun-like star will transit once every $\sim 1.5 \mathrm{yr}$, and the duration of each transit observation (including baseline measurements) would be up to $\sim 15 \mathrm{hr}$. Observing all of the planets with G-type hosts in Sample 3 during every transit would cost $\sim 1600 \mathrm{hr}$ ( $\sim 2$ months) per year; this commitment of time would be justified by the value of characterizing $>100$ "true" Earth twins.

However, planets orbiting low-mass stars are far more common with orbital periods of $<30$ days. The required amount of time for a transit observation is still significant (up to $\sim 5 \mathrm{hr}$ ), and to observe every transit of, for example, 500 such planets (Sample 2) would require $5-10 \times$ more observing time per year than is available. Fortunately, the short orbital periods and larger transit depths of such planets will allow an observer to achieve a high-S/N transmission spectrum with $\sim 10$ observations in less than a year, and the full sample of planets with M-type hosts could be studied sequentially over a decade.

Finally, since the $\mathrm{S} / \mathrm{N}$ scales with $\sqrt{N_{\mathrm{obs}}}$, there are diminishing returns when combining a large number (tens) of transit observations for a given target. Nevertheless, closer to $\sim 100$ visits could be scheduled for a handful of nearby interesting low-mass systems (e.g., TRAPPIST-1; Gillon et al. 2017) to enable very high $\mathrm{S} / \mathrm{N}$ spectroscopy, but we assume that the amount of time spent on such systems will be negligible in the overall scope of the mission. We use these arguments to set the number of combined transit observations for the results shown in Figure 6, and we see that in about $8 \mathrm{yr}$ we can achieve statistically significant positive results for the samples suggested in Table 1. 
Earth analog around Solar-type star at 100 parsecs

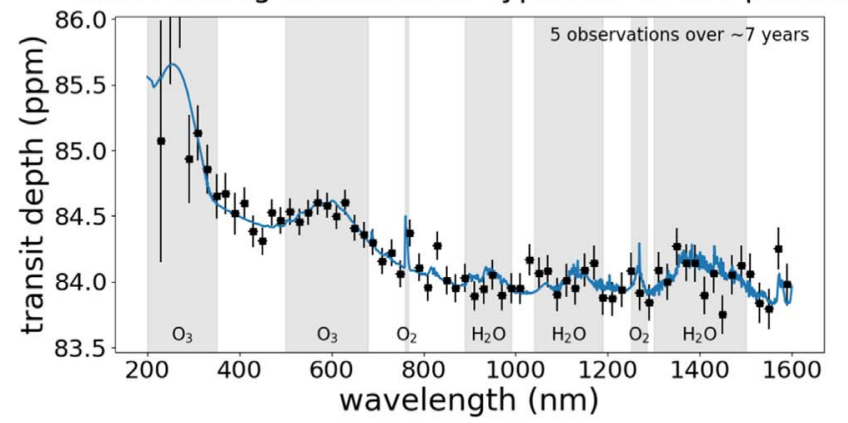

Earth analog around low-mass star at 10 parsecs

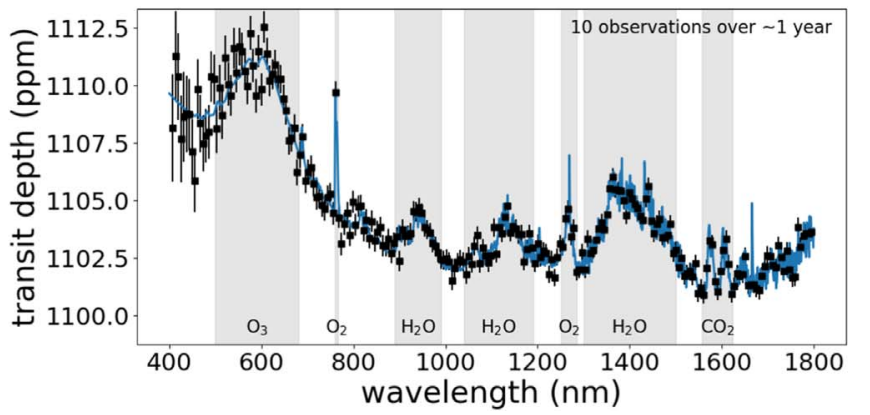

Earth analog around Solar-type star at 300 parsecs

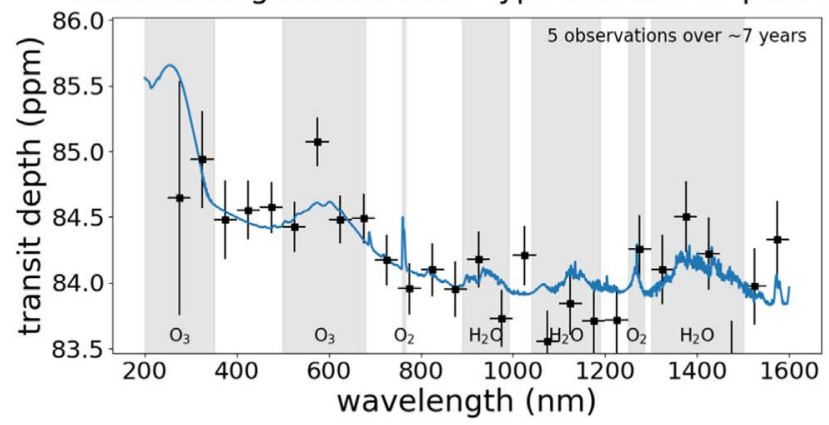

Earth analog around low-mass star at 50 parsecs

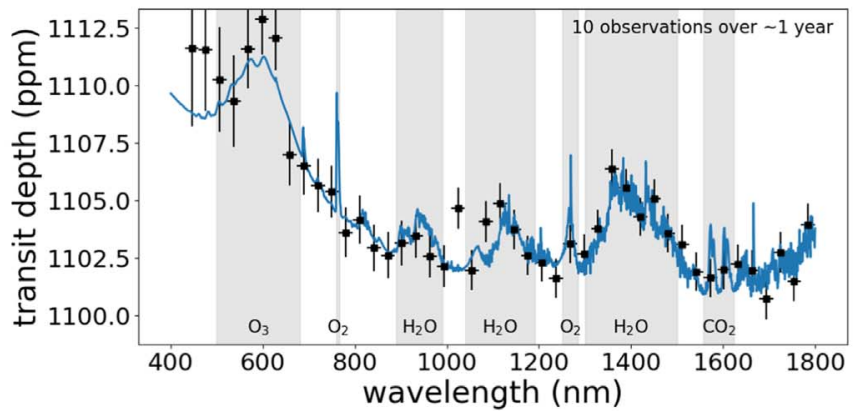

Figure 6. Simulated Nautilus observatory spectra of nearby and far targets (Earth-sized habitable zone planets) around low-mass stars (M dwarfs) and Sun-like stars ( $\mathrm{G}$ dwarfs), assuming 35 telescopes with $8.5 \mathrm{~m}$ diameter apertures. The light-collecting power equivalent to that of a $D \sim 50 \mathrm{~m}$ telescope enables the detection of $\mathrm{H}_{2} \mathrm{O}$ and $\mathrm{O}_{3}$ in $\sim 1000$ Earth analogs. The same configuration often also enables detection of $\mathrm{O}_{2}$ and, in hundreds of simulated Earth analogs around nearby low-mass stars, $\mathrm{CO}_{2}$ absorption.

Table 3

Baseline Science Requirements for the Nautilus Biosignature Survey

\begin{tabular}{lclr}
\hline \hline Parameter & Value/Range & Science Driver & \multicolumn{1}{c}{ Ref. } \\
\hline Num. exo-earth Candidates & 1000 & Expected exoplanet diversity, statistically meaningful results & Section 2.2 \\
Faintest host stars probed & $I=16.5$ & Furthest/coolest star in sample & Section 2.3 \\
Wavelength range & $0.45-1.0 \mu \mathrm{m}$ & $\mathrm{H}_{2} \mathrm{O}, \mathrm{O}_{3}$ molecular bands & Section 2.4 \\
Photometric precision & $\sim 1 \mathrm{ppm}$ & Absorption feature depth & Section 2.4 \\
Spectral resolving power & $\lambda / \Delta \lambda=50-170$ & Molecular bandwidths & Section 2.4 \\
\hline
\end{tabular}

\section{Preliminary Science Requirements and Operations}

For the purposes of this exploratory study we adopt the following high-level, preliminary science requirements: (1) an effective light-collecting aperture equivalent to a $D=50 \mathrm{~m}$ telescope; (2) a wavelength coverage that includes key biosignature absorption bands, such as $450-1000 \mathrm{~nm}$; (3) near photon-noise-limited telescope performance (including temporal stability) after post-processing; and (4) low-resolution spectroscopic capability with spectral resolution between 6 and $20 \mathrm{~nm}$ (corresponding to $R=\lambda / \Delta \lambda=50-170$ ).

In Table 3 we summarize the science requirements of a statistically meaningful atmospheric biosignature survey. In the following section (Section 3.1) we will describe our novel concept for a space telescope array capable of carrying out the biosignature survey described here. We note that these requirements are intended to be representative and not conclusive; future, more comprehensive studies will be required to fully define the requirements for an actual flight mission.

\subsection{Nautilus Telescope Array}

In this section we introduce the Nautilus observatory, a novel telescope array concept developed to meet the science requirements (see Section 3 and Table 3) of the large-scale atmospheric biosignature survey we described in Section 2. The Nautilus concept described here is not a complete, final, and fully optimized mission design. Rather, it is a notional design that highlights the potential of novel large-scale diffractive optics to answer key astrophysical questions. In this manuscript we focus on technical opportunities and challenges unique to MODE-lens-based space observatories or the Nautilus concept. We do not address technical aspects that are shared with other space observatories. We will first review the baseline concept for the observatory and its operations and then review individual unit telescope architecture, their launch and deployment, and various fundamental considerations.

\subsection{Nautilus Array Baseline Concept}

Our study described in Section 2.4 established that a telescope system with a light-collecting power equivalent to a single $50 \mathrm{~m}$ diameter aperture will be required for the biosignature survey. As no such large-diameter single telescope is realistic to launch in the foreseeable future, our mission design envisions multiple unit telescopes that combine light noncoherently to match the light-collecting power of a $50 \mathrm{~m}$ telescope. Our notional Nautilus concept utilizes an array of 


\section{a) 15 Unit Telescopes in SpaceX/BFR Fairing}

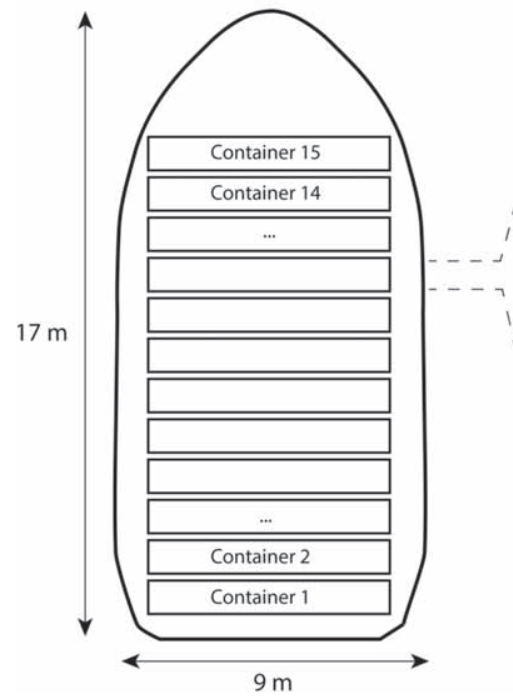

c) Deployed Unit, Side View

Thrusters for Angular Momentum Management
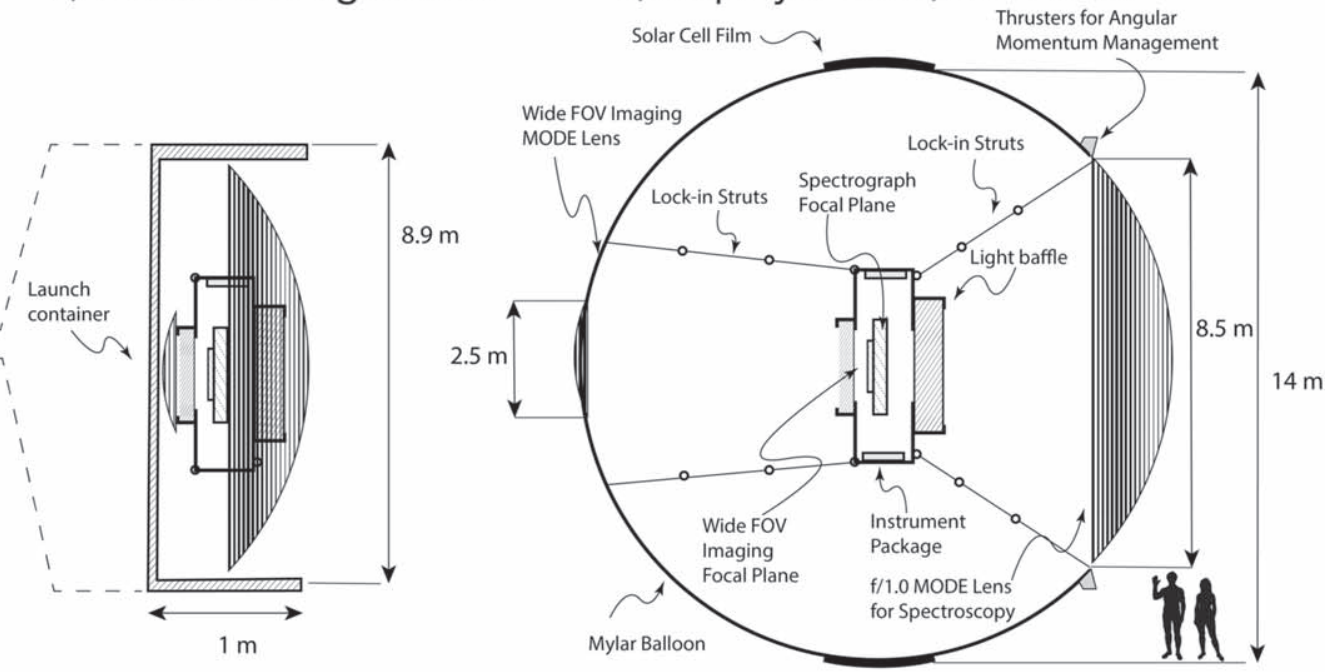

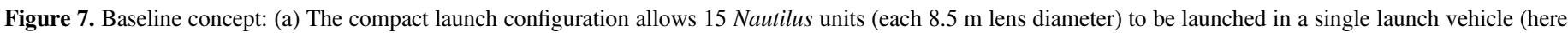

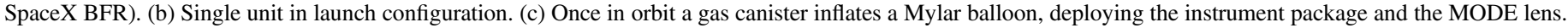

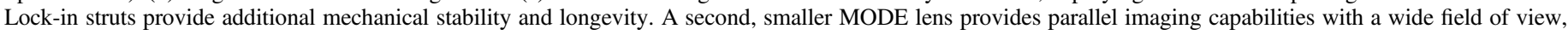
ideal for exoplanet transit search or deep imaging surveys.

ultralightweight, very large aperture, and low-cost unit space telescopes with powerful light-gathering capabilities. In considering the diameter of the individual unit telescopes, we adopted a size that is consistent with the largest rigid (nonfolding) diameter that can be launched in the next decades: specifically, we adopted a diameter of $D=8.5 \mathrm{~m}$, about $5 \%$ smaller than the maximum inner dynamic envelope diameter of the fairings of the next-generation vehicles (SpaceX/BFR, NASA SLS B2). In order to match the light-collecting power of a $50 \mathrm{~m}$ telescope, 35 such unit telescopes will be required. We note that the design presented here is not sensitive to the specific diameter of the unit telescope's diameter: if unit telescopes with smaller apertures are used, the number of unit telescopes can be increased to keep the total area constant. Future trade studies will be required to verify that the $35 \times 8.5 \mathrm{~m}$ configuration is an optimal choice.

This novel telescope architecture is potentially enabled by the rapid progress in replicated multiorder diffractive engineered material (MODE) lenses, which have the potential to replace primary mirrors. Their incoherently combined light (digitally co-added signal) collecting capability will equal that of a single $50 \mathrm{~m}$ mirror diameter space telescope.

We envision Nautilus to operate primarily in follow-up transit spectroscopy mode, but also to have the capability for exoplanet transit searches. The combined operations will allow discovering and characterizing a very large number of habitable zone Earth-sized transiting exoplanets.

Transit search mode: The Nautilus observatory will benefit from multiple powerful transiting exoplanet search missions that precede it (e.g., Kepler; Borucki et al. 2010; TESS; Ricker et al. 2015; PLATO; Rauer et al. 2014), which are expected to identify tens of thousands of transiting exoplanets. Nevertheless, the Nautilus observatory will be capable of searching for transits on its own, enlarging the potential target sample. Operating independently of each other, unit telescopes will monitor potential exoplanet host stars in the target sample, and through their parallel operation they will have the potential to carry out the most sensitive and most comprehensive transiting exoplanet search yet. The unit telescopes will use their smaller ( $2.5 \mathrm{~m}$ diameter) MODE lens-optimized for wide-field-ofview imaging-for the transit search. The transit search component will greatly expand the number of known transiting habitable zone Earth-sized planets.

Follow-up transit spectroscopy mode: During known transit events, all unit telescopes will record the transmission spectrum of the same planet using their larger, $8.5 \mathrm{~m}$ diameter MODE lenses. The signal measured by the individual unit telescopes will be combined noncoherently (by digitally co-adding), enabling the confident detection of major atmospheric absorbers $\left(\mathrm{O}_{2}, \mathrm{O}_{3}, \mathrm{H}_{2} \mathrm{O}\right)$ in Earth twins up to about $300 \mathrm{pc}$.

The noncoherent combination of signal, as planned in the Nautilus observatory concept, does not require formation flying for the unit telescopes. As light is combined noncoherently, the relative locations of the individual telescopes during the observations (as long as the target star is visible) are not important.

\subsection{The Nautilus Unit Telescope}

Figures 7-9 illustrate our baseline concept for the Nautilus unit telescopes in compact launch and deployed configurations. Figure 10 illustrates the observatory (telescope array) in operation. Each unit telescope will use an $8.5 \mathrm{~m}$ diameter $f / 1.0$ focal ratio MODE lens as the light-collecting element for the exoplanet transit spectroscopy observations, and a smaller, $2.5 \mathrm{~m}$ diameter, wide-field-of-view MODE lens for the photometric exoplanet transit search operations. The two lenses will focus the light on two simple instruments. Each Nautilus unit will be a stand-alone telescope equipped with two visual/near-infrared detectors and a low-resolution spectrograph optimized for the $0.45-1.0 \mu \mathrm{m}$ wavelength range.

In this study we will not address the details of posited instruments and detectors, as our focus is on the novel use of 


\section{Deployed Nautilus Unit Telescope}

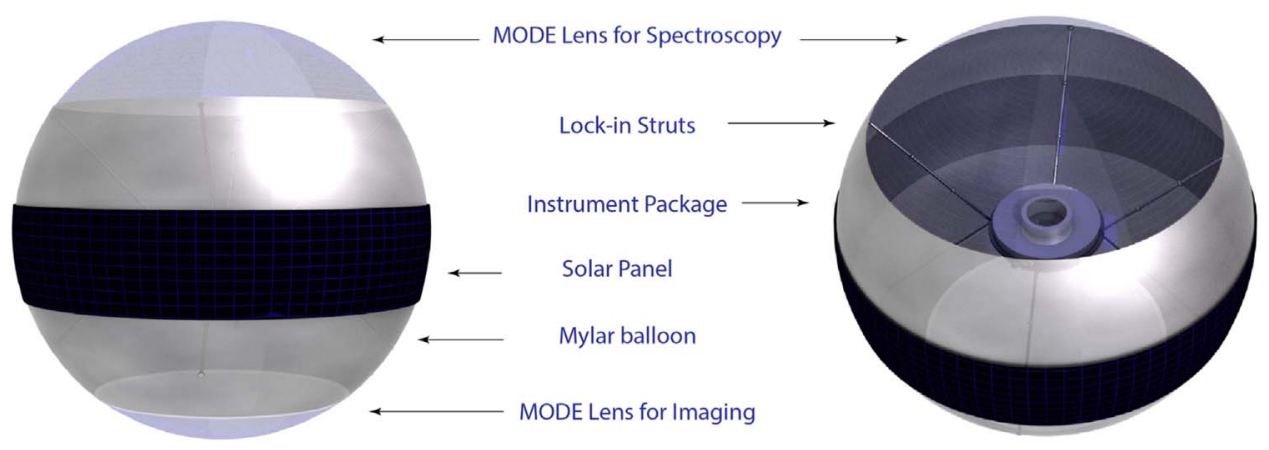

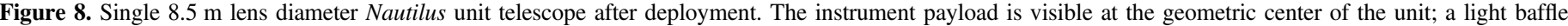
controls off-axis and internally scattered/reflected light. Lock-in struts provide mechanical stability. The solar cell film is visible as the equatorial dark belt.
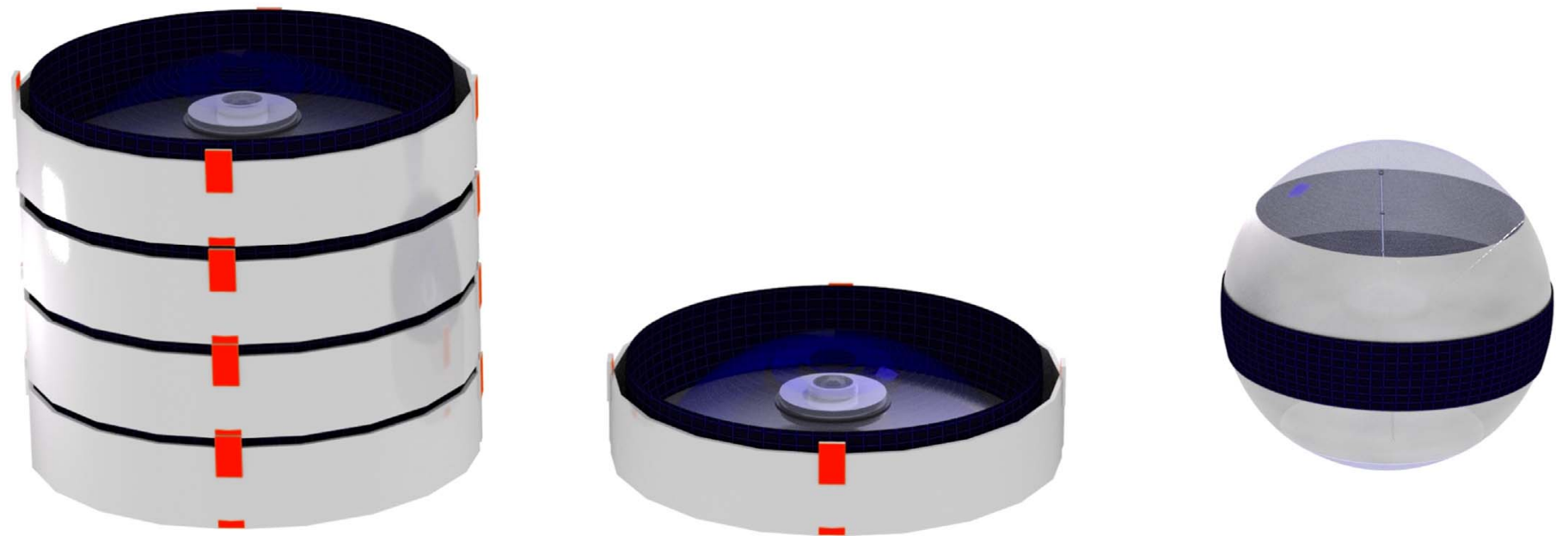

Figure 9. Rendering of the Nautilus launch containers (left), a single unit telescope in the launch container (middle panel), and a single deployed unit (right).

MODE lens technology and the measurements strategy it enables. We note, however, that the measurements proposed herein are generally compatible with low-noise detectors and low spectral resolution spectrographs that are currently available. If a sufficient amount of light is collected, currently available measurement technology offers viable solutions for the observations. For example, Kepler's CCD cameras were able to detect ppm-level modulations (after detrending, in white light) the same level of precision required by the observations proposed here. We also note that lower light throughput and detector noise can be compensated by increasing the number of unit telescopes, i.e., will not have a major impact on the overall concept.

\subsection{Orbit}

In this section we demonstrate that several satisfactory options exist for the orbit of the Nautilus observatory. The determination of the ideal orbit will be based on a detailed assessment of the mission concept; here we provide only a preliminary discussion. The primary considerations for the Nautilus array's orbit are low-energy $(\Delta v)$ access, orbital stability, a very stable thermal and radiation pressure environment, and quasi-constant illumination (from Sun, Earth, and Moon).
With these considerations in mind we identified the EarthSun L2 point as one of the possible locations for the Nautilus array. Stationed at the L2 point, the Sun, Earth, and Moon will be seen by the Nautilus units from nearly identical directions, allowing for a very stable radiation environment and constant communication windows. A possible alternative, easier-toaccess orbit would be a Sun-synchronous, high-inclination, terminator-following low Earth orbit. This orbit would provide somewhat less stable illumination and thermal environment and more limited sky coverage but would be easier to access and to communicate with. In addition, a low Earth orbit would enable passive angular momentum management (magnetic torquing rods). The ultimate choice of orbit will impact the spacecraft design.

\subsection{Launch and Deployment}

The Nautilus unit telescopes utilize inflatable spacecraft components for deployment, allowing the instrument package and MODE lenses to form very compact packages (see Figures 7-9). The unit telescopes are launched in a compact configuration, in cylindrical containers (approximately $9 \mathrm{~m}$ diameter and $1 \mathrm{~m}$ tall), with multiple containers positioned in a single fairing (Figure 9). Over the next two decades multiple launch options will exist that are suitable for the Nautilus observatory. Next-generation rockets and their largest fairing 

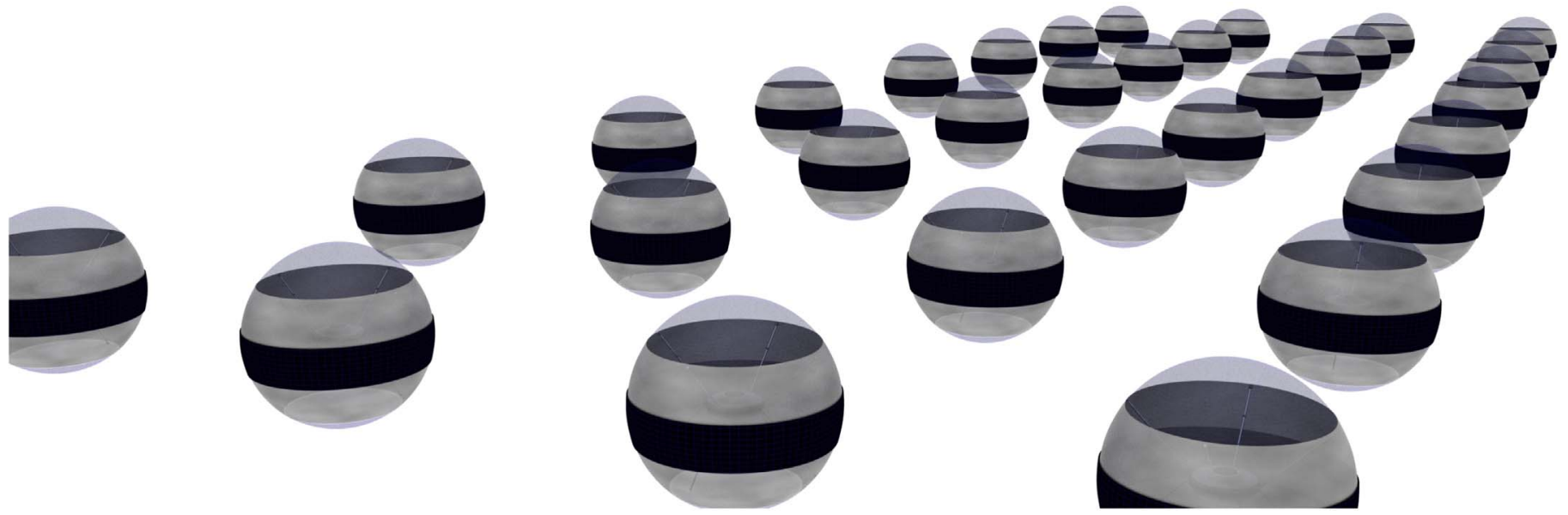

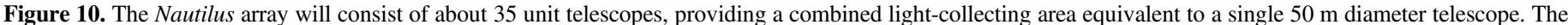
units do not need to fly in formation or even be located close to each other, as the intensity measurements are combined noncoherently (i.e., co-added).

will allow over a dozen units to be launched in a single payload (Figure 7): NASA's Space Launch System's Block 2B fairing is expected to offer a $\sim 9.1 \mathrm{~m}$ diameter payload envelope with a height approaching $30 \mathrm{~m}$; such a fairing may be capable of launching $\simeq 24-28$ Nautilus units in a single launch. SpaceX's upcoming Big Falcon Rocket (BFR) will offer a fairing with a diameter very similar to that of the SLS Block 2B long fairing, but with a probably shorter height $(17 \mathrm{~m})$. Correspondingly, SpaceX/BFR may be capable of launching 15 Nautilus units. In our reference design we adopt a BFR-style fairing (see Figure 7).

We note that currently existing fairings are well suited for launching smaller-but still very capable-pathfinder units. For example, the currently operational SpaceX Falcon 9 accommodates a dynamic payload envelope (cylindrical) with a $4.6 \mathrm{~m}$ diameter and $6.7 \mathrm{~m}$ height. This could accommodate up to six Nautilus launch modules with up to $\sim 4.2 \mathrm{~m} \mathrm{MODE} \mathrm{lens}$ diameter. The $5 \mathrm{~m}$ diameter "Long" fairing offered for the Atlas $\mathrm{V}$ has a fairing accommodating $4.6 \mathrm{~m}$ diameter with a $12.2 \mathrm{~m}$ height, which could be sufficient for 10 Nautilus units based on MODE lenses with diameters up to $\sim 4.4 \mathrm{~m}$.

After orbital insertion the individual Nautilus unit telescopes separate from the fairing and from each other and begin inflation. Mechanically, the telescope deployment is driven by the inflation of a spherical Mylar balloon (diameter $\sim 14 \mathrm{~m}$ ), which will shift the two MODE lenses (in front and behind of the instrument package) forward and backward by about $6.5 \mathrm{~m}$. In addition to the simple deployment mechanism, the Mylar balloon will also provide stray-light control, sunshield functionality, and solar energy to the telescope. The inflation itself is a nonreversible operation, initiated by the release of a low amount of chemically inert but relatively high atomic weight noble gas $(K r)$. Once the target shape is reached, mechanical struts lock in, fixing the telescope structure, providing long-term mechanical stability. The instrument packages will be aligned precisely to the focal plane of the deployed MODE lenses through observations of a reference star field.

\subsection{Power Source and Management}

The estimated power requirements of the Nautilus array unit telescopes are smaller than the power requirements of large space telescopes and therefore are not expected to pose a significant challenge. We anticipate that the major systems requiring energy will be communications, spacecraft attitude control, and the instrument package; similar components exists on HST, JWST, and Herschel Space Observatory, all powered via solar cell arrays.

As a baseline we estimate the operational power requirements of a Nautilus unit telescope by scaling the power use of $H S T(2.8 \mathrm{~kW})$ and JWST $(2.2 \mathrm{~kW})$. We anticipate that the power consumption of a single Nautilus unit telescope will be lower than HST and JWST owing to the Nautilus units' simpler design, the lack of cool instruments required for longwavelength observations $(>1.5 \mu \mathrm{m})$, its smaller number of instruments/subsystems, and considering more efficient electronics. As a reference value for power requirements we assume $1.5 \mathrm{~kW}$ for each telescope.

Unlike HST and JWST, Nautilus units will utilize flexible solar cell film, which has space heritage (Venus Express) anddue to the flexibility and low areal density-will provide ideal structural match for the inflatable spacecraft. Nautilus units will integrate solar cell film into the inflatable balloon in a rotationally symmetrical configuration. Assuming that the average fraction of the solar panels' surface illuminated is $\eta_{\text {ill }}=0.3$, a solar cell efficiency of $\eta_{\text {eff }}=0.1$, an average distance of $d=1$ au from the Sun, and a solar constant (at $1 \mathrm{au}$ ) of $c_{\text {Sun }}=1.37 \mathrm{~kW} \mathrm{~m}^{-2}$, the total surface area of Nautilus units covered in solar cell film will be $A_{\mathrm{sc}}=\frac{1.5 \mathrm{~kW}}{c_{\mathrm{Sun}} \eta_{\text {eff }} \eta_{\mathrm{ill}}}=36.5 \mathrm{~m}^{2}$ to provide $1.5 \mathrm{kw}$ average power. The power will be stored in batteries, providing a stable power source also when the solar array is not illuminated. The $36.5 \mathrm{~m}^{2}$ solar cell film corresponds to only about $6 \%$ of the spacecraft's (balloon's) surface (for a balloon radius of $7 \mathrm{~m}$ ). An orbit with greater average distance from the Sun will require a somewhat larger fraction of the balloon to be covered with solar cell film. In short, an available, low-weight and low-cost power source with space heritage exists that can, by a large margin, cover the energy needs of a unit telescope.

\subsection{Unit Telescope Instrument Package Volume}

Given the thin MODE lenses, we estimate the available instrument package volume as a cylinder $z=0.6 \mathrm{~m}$ high and with a radius of up to $r=4.0 \mathrm{~m}$, which translates into a volume of up to $\sim 30 \mathrm{~m}^{3}$. In comparison, HST's aft shroud is approximately $60 \mathrm{~m}^{3}$ (radius of $2.2 \mathrm{~m}$ and height of $3.55 \mathrm{~m}$ ). 
Therefore, a Nautilus unit telescope's instrument package volume would be overall comparable to instrument packages of existing major observatories. Given the goal to provide simple, compact, and identical instrumentation for each unit telescope, the volume available in the units is not expected to pose particular challenges.

\subsection{Angular Momentum Management}

Nautilus units will use four reaction wheels (all offset for the inertial axes) to manage the rotation (pointing, tracking) of the spacecraft along three axes (a fourth wheel provides redundancy). The reaction wheels will spin in the direction opposite to the intended rotation of the spacecraft. Nautilus units-like any spacecraft-will be subjected to net torques (primarily due to asymmetric exposure to solar irradiation and solar wind pressure). Although the reaction wheels will ensure stable pointing during operations, Nautilus units will periodically need to dump angular momentum. We envision two possible pathways for this: a passive and an active mechanism. In the passive angular momentum management mode - as the distribution of transiting planets is closely isotropic on the sky-each unit telescope's observing schedule can be planned in such a way to average out torques. In the active angular momentum management mode ambient-temperature, pressurized nitrogen is released through thrusters affixed to the exterior of the Mylar balloon (possibly at the connecting points of the lock-in struts; see Figure 7). Nitrogen does not affect the planned observations and will not react with or freeze onto the spacecraft. Given the unusually symmetric architecture of the unit telescopes, net torques will be lower than they are for most other spacecraft architectures, resulting in a much lower than typical rate of angular momentum accumulation.

\subsection{Pointing and Guiding}

The driver for the guiding stability is the high photometric precision: pointing drifts, combined with detector sensitivity variations and possible position-dependent systematics, will introduce apparent position-dependent intensity variations. While significant reduction in the power of such systematics is possible via post-processing (such as in the Kepler mission), it is desirable to keep image drifts at or below the level of the diffraction-limited spatial resolution of the telescope. Therefore, a guiding precision of approximately $15 \mathrm{mas} / 10 \mathrm{hr}$ would be targeted. The Nautilus unit telescopes will use the Sun as a coarse attitude reference point and the antisolar star field for precise pointing position measurements. The unit telescopes will use the target stars and reference stars within the field of view for fine guiding during long exposure series (typically $\sim 20 \mathrm{hr}$ ) before, during, and after planetary transits.

\subsection{Thermal Management}

At an orbit with an average distance of 1 au from the Sun, the spherical Nautilus units may operate close to room temperature $\left(25^{\circ} \mathrm{C}\right)$ with only modest active thermal management (heating). The inflatable balloons of the Nautilus units will protect the instrument package (in the interior) from large temperature excursions, as the nitrogen gas and emission/absorption within the balloon redistribute heat. Nevertheless, the high-precision measurements require a thermally stable system (instrument package, lens alignment, and lens itself). Therefore, Nautilus units will actively control the temperature of the elements within the spacecraft and of the MODE lenses. Heating will be provided by battery-powered thermoelectric cells, and excess heat will be dumped at the dark (nonilluminated) side of the Nautilus units, possibly through a metal ring surrounding the MODE lens (as MODE lenses are not exposed to the Sun during normal operations).

\section{Design, Fabrication, and Scaling}

In this section we review the current status, challenges, and pathways for the optical design and fabrication of large MODE lenses the Nautilus array concept is based on. This discussion is followed by a summary of the design challenges for the spacecraft architectures.

\subsection{MODE Lens Fabrication Process}

Due to their noncontinuous surface microstructures, MODE lenses cannot be fabricated through traditional grinding and polishing methods. Potential fabrication for noncontinuous surfaces includes diamond turning and molding, gray-scale lithography, deep-reactive ion etching, UV imprinting, and glass slumping. Among these, diamond turning and molding are the most powerful approaches for MODE lens fabrication owing to their accuracy and scalability. The combination of diamond turning and pressure molding offers very powerful and flexible fabrication paths for MODE lenses: diamond turning enables precise fabrication, and pressure molding enables reliable and low-cost replication.

Ultraprecision diamond-turning machines have been successfully used to fabricate conventional lenses and diffractive optical elements (e.g., Lee \& Cheung 2003; Huang \& Liang 2015). For example, state-of-the-art Moore Nanotech 350FG free-form generators are capable of diamond turning or milling MODE lenses or MODE lens segments with diameters up to $0.6 \mathrm{~m}$. Precision glass compression molding is a replicative process that allows the production of high-precision optical components from glass and polymer (Zhang \& Liu 2017), including those of diffraction surfaces (Huang et al. 2013; Nelson et al. 2015). By using chalcogenide glasses, precision molding also allows replicating optical elements for infrared applications (Staasmeyer et al. 2016). Compression molding has been successfully used to mold glass free-form optics from diamond-machined molds (He et al. 2014).

By combining diamond turning/milling to fabricate molds and glass press molding, it is thought to be possible to replicate large-aperture MODE lenses. Our team is actively developing this technology and fabricated, replicated, and tested lens prototypes. To illustrate the fabrication approach, we diamondturned molds and molded the MODE lens from poly(methyl methacrylate). One such prototype is shown in Figure 11. The measured lens profile and image quality verified the molded surface shape and quality. Our University of Arizona-based team is developing this technology toward very large aperture MODE lenses that can be replicated reliably and at low cost.

\subsection{MODE Lens Prototypes}

As part of the MODE lens development effort at the University of Arizona, our team has designed and fabricated several generations of diffraction-based lenses, from singleorder to more complex $(M=1000)$ MODE lenses. The latest prototype MODE lens combined a high-order diffractive lens 

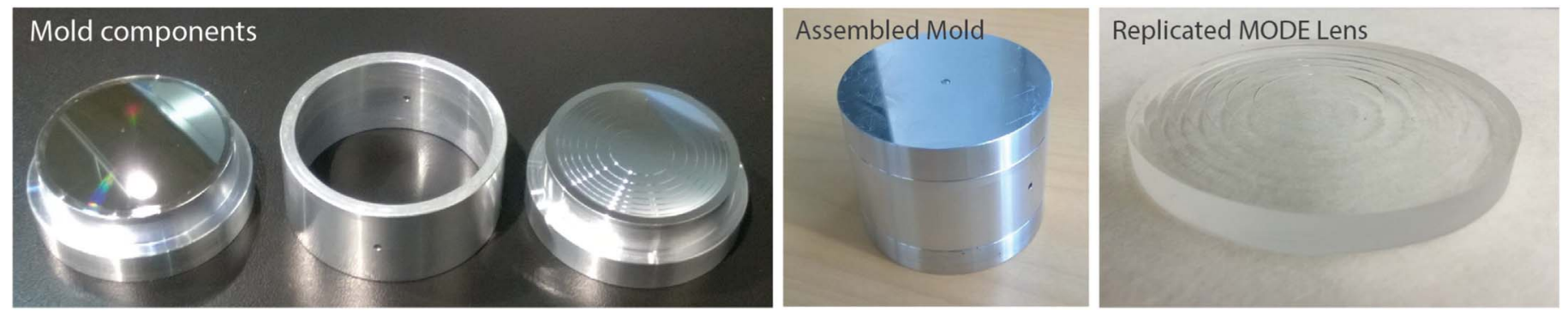

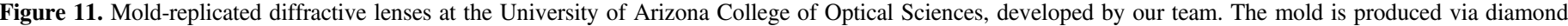

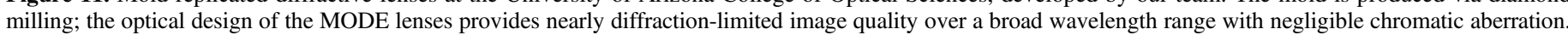
The MODE lenses can be readily and very cost-effectively replicated.

$(M=1000)$ at the front surface with a single-order Fresnel lens on its back surface. The optical design has been optimized to provide diffraction-limited performance in the astronomical $R$ band $(589-727 \mathrm{~nm})$. Laboratory optical tests with supercontinuum laser and imaging tests demonstrated that the measured performance is consistent with that predicted by the physical optical models (T. Milster et al. 2019, in preparation). Prototypes equipped with additional, conventional colorcorrector optics (much smaller in diameter than the MODE lens) are predicted to provide diffraction-limited performance over a broad wavelength range $(>200 \mathrm{~nm})$.

\subsection{Optical Quality Assessment}

In order to manufacture, align, and assemble a large MODElens-based space telescope system, specialized metrology concepts and solutions testing and verifying its optical performance need to be developed and applied. The challenging science goals of next-generation space telescopes are often achieved through a new optical design and components, such as a MODE lens. Measuring and aligning those complex optical surfaces and components require a large dynamic range in metrology, high accuracy, comprehensive spatial frequency coverage, and real-time data acquisition and analysis to test the multiscale optical features. Unlike the traditional small-size diffractive optical component, the diffractive optical surface of the MODE lens has a precision microscopic surface profile over its large-aperture area, enabling its light-collecting power. As stray light and surface scattering must be controlled for high-quality imaging performance, the nanoscale surface roughness must be measured and controlled. In order to realize the next-generation optical systems producing a near-optimal point-spread function with superb imaging quality, an entire spectrum of the optical system's wavefront must be measured and confirmed during the telescope manufacturing, assembly, and testing process.

As the entire spatial frequency spectrum of the optical surface/wavefront errors has to be controlled, the MODEbased space telescope optics need to be modeled and specified using a power spectral density (PSD) or structure function (Hvisc \& Burge 2007; Parks 2010). Parks (2008) experimentally demonstrates the severe image quality degradation due to the presence of intermediate to high spatial frequency surface errors.

Phase-shifting deflectometry is applied to measure, align, assemble, and evaluate the performance of a large optical system with nanometer-level accuracy through its direct slopemeasuring capability ( $\mathrm{Su}$ et al. 2010; Oh et al. 2016). During fabrication, the quality of the MODE lens local surface finish (i.e., microroughness rms value) is monitored and sampled across the large aperture using a portable white-light interferometer (Parks 2011). Various metrology systems covering different ranges of spatial frequencies measure and test the MODE-based space telescope by providing a comprehensive PSD evaluation similar to the $4.2 \mathrm{~m}$ Zerodur primary mirror of the Daniel K. Inouye Solar Telescope (DKIST) tested with a suite of metrology systems as shown in Kim et al. (2016).

Other key components of the space telescope system utilize aspheric optics to achieve better achromatic imaging performance for a larger field of view within a compact and lightweighted design. For most cases, temporal phase-shifting interferometry uses a null component such as computergenerated holograms that provides high-accuracy wavefront/ surface measurement data with sufficient spatial frequency sampling. During the manufacturing and aligning process, in order to guide the processes, high dynamic range metrology methods are utilized. The wide range ensures the measurement of the optical component's or system's quality when it is still far away from its final specification performance. For instance, the 4-8 $\mathrm{m}$ diameter class high-precision optics manufacturing process and final testing of the Giant Magellan Telescope and DKIST primary mirrors were guided with a successful rapid convergence by utilizing nonnull deflectometry measurement feedback (Su et al. 2012; Huang et al. 2015).

For the alignment of MODE lens systems (as also applicable for active/adaptive wavefront correction or bending mode measurements) instantaneous metrology is applied using a multiplexed deflectometry solution. The real-time metrology system uses a multiplexed color fringe pattern in $x$ and $y$ spatial frequency domain in order to contain and process six fringe patterns from a single-shot data acquisition offering about $25 \mathrm{~nm}$ rms accuracy (Trumper et al. 2018). Such a dynamic measurement and active characterization of the MODE lens telescope system will monitor and verify the opto-mechanical performance as a function of time, orientation with respect to gravity, and thermal gradient changes. An overview of the baseline MODE lens optical specification as a function of spatial frequency (i.e., cycles per aperture) is given in Table 4.

\subsection{Optical Performance}

As an indication of system optical performance, spot diagrams are calculated that show geometrical ray intercepts at the image plane. These diagrams trace bundles of rays through the optical system from a point source (like a star) at a large distance. Figure 12 shows spot diagrams of a $0.24 \mathrm{~m}$ prototype MODE lens telescope at an intermediate image plane after color correction. The location of the idealized 1st Airy 


\section{Polychromatic Spot Diagram at Virtual Image Plane for Color-corrected MODE lens}

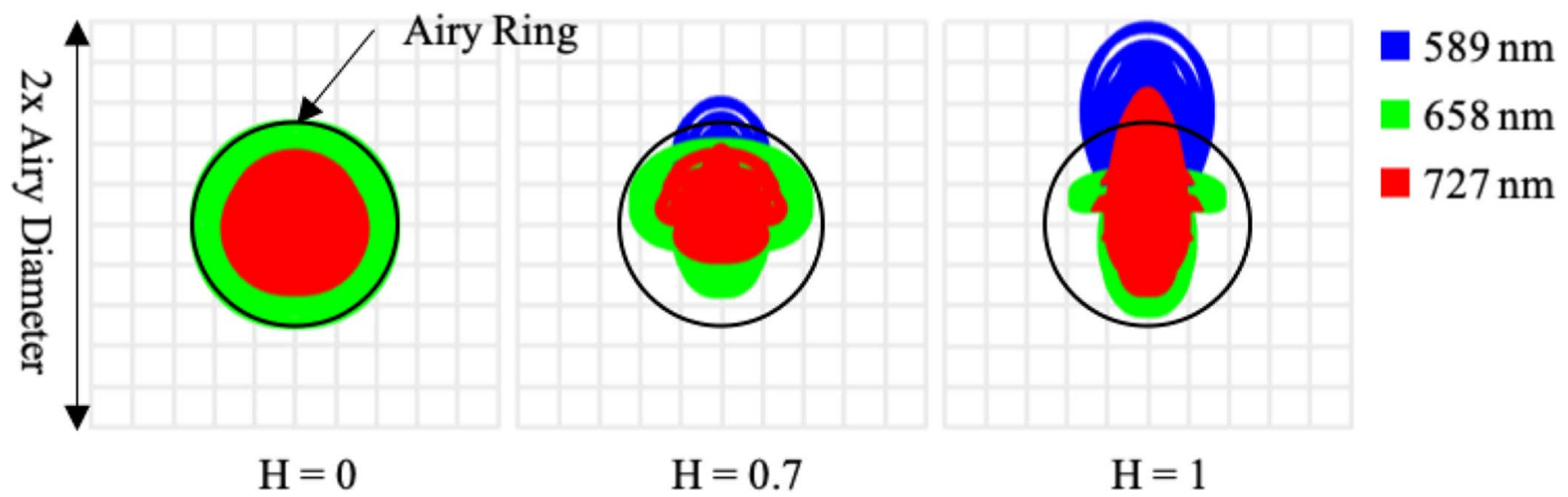

Figure 12. Polychromatic spot diagram at the virtual image plane of the color-corrected MODE telescope for an existing $0.24 \mathrm{~m}$ diameter design. In this design the Airy diameter is $2.94 \mu \mathrm{m}$ and the maximum full field of view $(H=1)$ is $7.5 \mathrm{arcmin}$. The image performance is diffraction limited on-axis $(H=0)$, and the spot size is only slightly larger than the diffraction limit at the edge of the field of view $(H=1)$.

Table 4

Overview of the Spatial Frequency Optical Specification for the MODE Lens at Wavelength $\lambda$

\begin{tabular}{|c|c|c|c|}
\hline Specification & Spatial Frequency & Requirements & Note \\
\hline $\begin{array}{l}\text { Transmitted wavefront error due to the surface } \\
\text { figure error }\end{array}$ & Low ( $<4$ cycles per aperture $)$ & $<\sim 0.017 \lambda \mathrm{rms}$ & $\begin{array}{l}\text { Interferometric measurement using } \mathrm{HeNe} \text { laser } \\
(\lambda=633 \mathrm{~nm})\end{array}$ \\
\hline $\begin{array}{l}\text { Transmitted wavefront error due to the inter- } \\
\text { mediate to high spatial frequency errors }\end{array}$ & $\begin{array}{c}\text { Medium (4-60 cycles per } \\
\text { aperture) }\end{array}$ & $<\sim 0.015 \lambda \mathrm{rms}$ & $\begin{array}{l}\text { Combining interferometric and deflectometric } \\
\text { measurements }\end{array}$ \\
\hline $\begin{array}{l}\text { Transmitted wavefront error due to the inter- } \\
\text { mediate to high spatial frequency errors }\end{array}$ & $\begin{array}{c}\text { High ( }>60 \text { cycles per } \\
\text { aperture) }\end{array}$ & $<\sim 0.017 \lambda \mathrm{rms}$ & $\begin{array}{l}\text { Combining interferometric and deflectometric } \\
\text { measurements }\end{array}$ \\
\hline $\begin{array}{l}\text { Surface roughness error due to the microsurface } \\
\text { finish }\end{array}$ & $\begin{array}{l}\text { Very high (sampling resolu- } \\
\text { tion }<100 \mu \mathrm{m} / \text { cycle) }\end{array}$ & $<\sim 2 \mathrm{~nm} \mathrm{rms}$ & $\begin{array}{l}\text { Measurement using white-light interferometer over } \\
1 \times 1 \mathrm{~mm} \text { area with } 500 \times 500 \text { sampling points }\end{array}$ \\
\hline
\end{tabular}

ring minimum is shown as a black circle. The Airy pattern is the image-plane light distribution due to diffraction of the optical system illuminated by a distance point source. The diameter of the Airy ring is dependent on the wavelength and $f$ number. In this case, the Airy ring is calculated from the prototype system parameters with a central wavelength of $\lambda_{c}=658 \mathrm{~nm}$ and an $f$-number of 1.83 . The image is considered diffraction limited if all of the geometrical ray intercepts fall within or near the Airy ring. In Figure 12, the geometrical ray intercepts are calculated from three wavelengths of the point source, 589, 658, and $727 \mathrm{~nm}$, which are shown as blue, green, and red colors, respectively. Calculations are also made from three field angles of the point object, from on-axis (at $H=0$ ) to a maximum field angle of $7.5 \operatorname{arcmin}(H=1)$. The prototype design is diffraction limited on-axis at $H=0$ and out to $70 \%$ of the maximum field of view $(H=0.7)$. It is nearly diffraction limited at the maximum field of view $(H=1)$. Degradation of the system performance with increasing field angle is due to residual wavelength-dependent aberrations. While this design shows a 0.24 m diameter MODE-lens-based telescope system's performance, similarly diffraction-limited performance can be achieved by larger systems.

\section{Discussion}

The Nautilus mission concept described here envisions a space telescope array based on low-cost, replicated, $8.5 \mathrm{~m}$ diameter inflatable space telescopes utilizing novel, ultralight diffractive optics. Nautilus and the enabling technology will transform the design, construction, operation, and launch of space telescopes for scientific, commercial, and other applications. In the following we briefly compare MODE-lens-based technology and, specifically, the Nautilus design to the state of the art in space telescopes, and we review the primary advantages of the MODE technology over current mirror-based approaches.

\subsection{Comparison of Capabilities to the State of the Art}

No existing telescope is capable of searching for atmospheric biosignatures in exoplanets. JWST may be able to search for water and methane in the most favorable transiting exoplanets around very nearby red dwarf host stars, but probably in not more than two to four habitable zone Earth-sized planets. The mission concepts HabEx and LUVOIR would utilize highcontrast direct imaging to study Earth-like planets; they may image 50-300 planetary systems and search for biosignatures in up to about 10 to $\sim 60$ habitable planet candidates, respectively.

In contrast, Nautilus is a system concept developed to survey $\sim 1000$ Earth-like planets, providing more than an order-ofmagnitude increase over the capabilities of even the most ambitious missions yet studied. The proposed survey can be accomplished with an array of telescopes that combine light incoherently. Achieving this level of light collection in a single, 
phased aperture is not a realistic possibility for the foreseeable future.

In terms of light-gathering power Nautilus offers an ordersof-magnitude increase over current facilities. Currently the largest-diameter space telescopes are $\operatorname{HST}(D=2.4 \mathrm{~m})$ and Herschel $(D=3.5 \mathrm{~m})$, with the $D=6.5 \mathrm{~m} J W S T$ soon to follow. A single element of the Nautilus concept will exceed HST's light-gathering power by a factor of 12.5, and the Nautilus array will exceed JWST's collecting area by a factor of 60 (The LUVOIR Team 2018).

\subsection{Tolerance to Misalignments}

Unlike reflecting telescopes, our MODE lens design is inherently more tolerant of optical element misalignments, a fact that will significantly reduce fielding costs (e.g., Lo \& Arenberg 2006). If a mirror orientation is tilted by angle $\alpha$, the reflecting beam will be deflected by $2 \alpha$, which requires a very tight alignment tolerance and complex control solutions. However, with a basic first-order geometrical optics analysis, a chief ray going through the center of a refractive lens does not change its direction, although the lens is tilted. In a similar manner, transmissive refractive/diffractive optics are insensitive to surface figure errors, including intermediate to high spatial frequency errors. For example, an anomaly with height $h$ on a mirror surface in space will induce $2 h$ optical path length (OPL) change owing to its double-path nature. However, for a lens with a refractive index of $n$, the same surface anomaly will cause only an $(n-1) \times h$ OPL difference, i.e., only a $\sim 0.5 \mathrm{hr}$ change in OPL (assuming a typical $n=1.5$ ). Also, if the thin MODE lens is bending or locally rippling while it is maintaining the thickness of the MODE lens, there is almost no OPL change since the front and back surfaces are moving together. This robustness of the alignment and shape error tolerance is one of the most fundamental strengths of the MODE-lens-based telescope system.

\subsection{Mass Comparison to Mirrors}

As an illustration of the anticipated mass advantage of MODE-based space telescopes over reflecting space telescopes, we contrast scaled-up versions of the HST and JWST mirror systems with MODE telescopes. HST uses a 2.4 m diameter, $33 \mathrm{~cm}$ thick, monolithic mirror, whose weight is reduced with respect to a conventional monolithic mirror by about a factor of five through the implementation of a honeycomb structure in the body of the mirror. The mass of HST's mirror is approximately $826 \mathrm{~kg}$, which corresponds to about $7.4 \%$ of the total observatory mass. The HST mirror provides an excellent reference point for relatively lightweight monolithic space telescope mirrors.

JWST is a primarily infrared telescope with diffractionlimited optical performance at and beyond $2 \mu \mathrm{m}$. It utilizes 18 gold-coated beryllium mirror segments, each of which is $20.1 \mathrm{~kg}$. The segments are periodically co-phased between observations. Considering the mirror control structure (wire harness), the complete primary segment assembly for each mirror segment is $39.48 \mathrm{~kg}$. This leads to a combined mass of $710 \mathrm{~kg}$ for the $6.5 \mathrm{~m}$ mirror, corresponding to $9.6 \%$ of the total observatory mass. JWST is the natural reference for state-ofthe-art segmented space telescope mirrors.

In Table 5 we compare the masses of hypothetical $8.5 \mathrm{~m}$ diameter mirrors that use $H S T$ - and JWST-like mirror systems.
Table 5

Mass Comparison between Different Mirror and MODE Lens Primaries

\begin{tabular}{|c|c|c|c|c|}
\hline Case & $\begin{array}{c}H S T \\
\text { Honeycomb }\end{array}$ & $\begin{array}{c}J W S T \\
\text { Segmented }\end{array}$ & $\begin{array}{c}\text { MODE } \\
0.5 \mathrm{~cm}\end{array}$ & MODE $5 \mathrm{~cm}$ \\
\hline $\begin{array}{l}\text { Current } \\
\text { Design }\end{array}$ & $2.4 \mathrm{~m}, 826 \mathrm{~kg}$ & $\begin{array}{l}6.5 \mathrm{~m}, \\
710 \mathrm{~kg}\end{array}$ & $\cdots$ & \\
\hline \multicolumn{5}{|c|}{ Assuming $M \propto D^{2.0}$ scaling for HST and JWST: } \\
\hline $\begin{array}{l}\text { Scaled } \\
\quad \text { to } 8.5 \mathrm{~m}\end{array}$ & $10,360 \mathrm{~kg}$ & $1,214 \mathrm{~kg}$ & $425 \mathrm{~kg}$ & $4,255 \mathrm{~kg}$ \\
\hline \multicolumn{5}{|c|}{ Assuming $M \propto D^{2.8}$ scaling for HST and JWST: } \\
\hline $\begin{array}{l}\text { Scaled } \\
\quad \text { to } 8.5 \mathrm{~m}\end{array}$ & $28,494 \mathrm{~kg}$ & $1,505 \mathrm{~kg}$ & $425 \mathrm{~kg}$ & $4,255 \mathrm{~kg}$ \\
\hline
\end{tabular}

Note. Current and Nautilus-like diameters listed. For the $8.5 \mathrm{~m}$ diameter HSTand JWST-like mirror systems the masses of the mirrors are scaled with their diameters following the two scaling relationships indicated. The mass of the MODE lens is calculated from its volume and typical glass density $\left(2500 \mathrm{~kg} \mathrm{~m}^{-3}\right)$. Two different thicknesses are shown for the MODE lens to illustrate anticipated mass range. MODE lenses have the potential to provide low-cost and very low weight alternatives to mirror systems, with extrapolated mass well below HST-like honeycomb mirrors and similar to JWST's ultralight segmented mirror system.

We provide two bracketing cases for the mass scaling with diameter: an optimistic case when the mass $M$ is directly proportional to the $D$ collecting area of the mirror $\left(M \propto D^{2.0}\right)$, and a more conservative one $\left(M \propto D^{2.8}\right)$ in which the larger area also translates into a thicker mirror (or additional cophasing system). We compare these extrapolated mirror masses to two MODE lenses: a thin ( $h=0.5 \mathrm{~cm}$ thick, optimistic case) and a thick ( $h=5 \mathrm{~cm}$ thick, pessimistic case) lens. We note here that the thickness of the MODE lens will be likely set by mechanical structural considerations and not the optical design, as even few-millimeter-thick MODE lenses can provide excellent image quality. To calculate the mass of the MODE lens (see Section 1.3), we consider its volume $\left(R^{2} \pi \times h\right)$ and the volume-filling factor $\phi=0.5$ and assume a glass density of $\rho=2500 \mathrm{~kg} \mathrm{~m}^{-3}$.

Table 5 demonstrates that the MODE lens technology is expected to be a factor of 2 (worst case) to 70 (best case) lighter than an HST-like honeycomb mirror. Compared to JWST-like segmented mirrors, MODE lenses have similar mass (between 3 times lighter and about 3 times heavier). Compared to a conventional mirror (about 5 times more massive than honeycomb mirrors), MODE lenses would provide about two orders of magnitude lower mass.

Not only will MODE technology enable ultralight lightcollecting capability (on par with or better than the lightest mirrors), further significant reduction in the total mass for a MODE-based telescope system is expected given the overall lighter support structure required and owing to the fact that the MODE lenses are much more tolerant to misalignments.

\subsection{Potential for Lower Launch Costs}

The Nautilus concept benefits from a potential for greatly reduced launch costs through four factors: First, MODE-lens-based telescopes will be much lighter than telescopes based on monolithic mirrors and about as light as the lightest segmented mirror systems (Section 5.3). Second, with the typically twoorders-of-magnitude more relaxed alignment tolerances (Section 5.2), the structural support requirements are milder and 
Table 6

Assessment of Elements of Cost

\begin{tabular}{|c|c|c|}
\hline Element of Cost & Traditional Approach & MODE Relative Cost \\
\hline Design & Same & Same \\
\hline Materials & Larger owing to the greater mass of materials. Structure more costly than & Lower \\
\hline Manufacturing tooling & Larger owing to the need for more types of machines & Lower \\
\hline Manufacturing recurring cost & $\begin{array}{l}\text { Larger owing to greater time to produce an optic; each step is longer in time than the MODE } \\
\text { molding step }\end{array}$ & Lower \\
\hline Alignment, integration, and testing & Larger owing to the need to integrate mirrors into structure & Lower \\
\hline Verification & Same & Same \\
\hline
\end{tabular}

allow for the use of lightweight structural elements, such an inflatable deployment mechanisms (Section 3.1). The lightweight and inflatable structural elements represent significant further reductions in the telescope's mass. Third, MODE lens systems can provide simultaneously fast systems (small focal ratios) and wide fields of view. This allows the MODE telescopes to be very compact ( $f / 1.0$ systems), thus alleviating the need for light path folding and secondary mirrors. Fourth, the very compact launch configuration and low weight enable the simultaneous launch of many unit telescopes in a single launch fairing (e.g., up to 15 with SpaceX/BFR or 25 with NASA SLS B2 Long). This dramatically reduces the per-telescope launch costs. The Nautilus concept is too preliminary to allow for reliable costing. It seems, however, that due to the four factors discussed above, MODE-lens-based systems - and, in particular, the Nautilus observatory-have the potential to provide a significantly lower launch cost solution than those following a more conventional design.

\subsection{Potential for Lower Mission Costs and Risks}

It is argued that current mission costs and complexity are driven by the so-called "space spiral," in which higher reliability requires a longer development phase, which results in fewer missions and higher mission costs, which-in turnrequires even higher reliability (Wertz et al. 2011). This selfreinforcing cycle arguably drove mission costs, and it is estimated that at present day the average cost of space systems launched by the US is about 3 billion USD per launch (Wertz et al. 2011).

The Nautilus observatory represents a new approach to space telescope fabrication, one in line with the development of small satellites and diversified launch capabilities. The Nautilus concept has the potential to reduce mission costs in three major ways: First, it utilizes a low-cost (replicated) optical element instead of massive and complex mirror systems. This reduces the fabrication cost of one of the primary components of telescopes that conventionally drives mass, risk, and cost budgets. This represents a fundamental paradigm shift, making the production of space telescopes far more economical. Second, the MODE lenses provide ultralightweight alternatives to mirrors and enable lightweight telescope structures also utilizing inflatable elements, translating into a major reduction in launch costs (Section 5.4). Third, unlike many past space telescopes and space observatories, Nautilus is envisioned not as a unique, high-reliability, and very expensive telescope but as an array of replicated, identical, relatively low cost telescopes. This major difference is enabled by the ability to efficiently replicate the key optical element by using the MODE technology.
The model in which many unit telescopes are built and launched also alleviates the very high reliability requirement: compromised operation (or even failure) of one unit telescope would not compromise the array's overall capabilities. Similarly, the instruments envisioned for Nautilus units are simple and replicated, less capable than typical HST and JWST instruments, but also with individually relaxed fault tolerance, and they can be built for a fraction of the cost. In addition, the risks could be better distributed than possible in the current single, unique mission model: we can envision the launch of one or two smaller-size demonstrator units to mitigate risks.

The specific cost of the MODE lens and its associated system cannot be rigorously derived at this early TRL and mission concept maturity level (CML). However, the technology offers a likely cost advantage over traditional systems, and a qualitative argument can be provided to establish that MODE lenses are less expensive than traditional approaches. Let the cost of each stage $i$ in the development of the MODE and traditional optics be denoted as $M_{i}$ and $T_{i}$, respectively. Correspondingly, the total cost of a MODE-based system is $\Sigma_{i} M_{i}$, while the total cost of a traditional mirror-based system is $\Sigma_{i} T_{i}$

Since at this early system TRL and CML we cannot reliably predict total cost, we use another method of analysis. This method is a heuristic one and shows that $M_{i} \leqslant T_{i}$ for all $i$. Consider Table 6, which lists the assessment at each stage of why the traditional approach is likely to be equal to or more costly than the MODE technology.

As shown in Table 6, our assessment is that for each step the cost for the MODE lens is less than or equal to the cost of traditional methods, meeting the condition that $\Sigma_{i} M_{i}<\Sigma_{i} T_{i}$, and therefore indicating the cost efficiency of the MODE technology. As the technology and concept develop, we will be able to improve on the originally heuristic cost argument more quantitatively and precisely, but the qualitative result given is likely not to change.

\subsection{Scalability and Scalability Challenges}

Due to its relatively low production and launch costs and the identical multispacecraft model that is relatively new to astrophysical space telescopes, the general Nautilus system proposed here provides an easily scalable approach. Such multispacecraft models (multiple identical units) are used commercially (Iridium system) and for geo- and planetary sciences (Voyagers, Mariners, Mars exploration rovers, etc.) to reduce per-unit costs and risks and to extend capabilities. Furthermore, telescopes utilizing similar architecture but 
increasing in size could demonstrate feasibility and mitigate risks, while producing scientific data.

As discussed in Section 4.1, the combination of optical highprecision diamond turning and compression molding has a clear potential for enabling the efficient replication of very large scale diffractive optical elements. In Section 4.3 we reviewed considerations for optical quality monitoring for such large-scale optical elements and showed that the required technology already exists today and that only minor changes will be required to adopt it for MODE lenses.

Here we will briefly review three challenges that must be overcome to enable the replication of large-scale MODE lenses. First, diamond-turning and molding machines must be scaled up significantly. Both of these technologies are fundamentally not very sensitive to spatial scales, i.e., the construction of large diamond-turning and molding machines is thought to be entirely possible by just building larger versions of the current machines. No change in technology is required.

Second, the structural integrity of the large-diameter MODE lenses must be preserved during launch (unless molded in space). Large-scale, relatively strong, yet thin transmissive glass elements exist in a variety of fields. For example, car windshields (layered glass panels) are large diameter and strong, yet typically only $4-6 \mathrm{~mm}$ thick. Nevertheless, the fabrication, handling, and launch of very large, lightweight optical glass elements will clearly represent a challenge. Third, the deployment of the large MODE lenses through a lightweight structural element (such as a balloon; Section 3.1) must be demonstrated. Inflatables and optical deployables have a long heritage in satellites (e.g., ECHO-1, or solar panels), and heritage solutions may already exist for smaller scales. The fact that MODE lenses are very tolerant of misalignments (deployment errors) is encouraging. Although there are reasons to believe that all three of these challenges are surmountable in the very near future, MODE lens technology development and mission concept design must mitigate these risks.

\subsection{Science Impact}

MODE-lens-based, very large aperture telescopes in general, and the Nautilus concept specifically, offer a possible revolution in the light-gathering power of astronomical space telescopes. The greatly enhanced light-gathering power equals greatly enhanced sensitivity to faint astrophysical objects (such as the earliest, very high redshift galaxies; supernovae at high redshifts; individual stars in nearby resolved galaxies; or small minor bodies in the solar system). The enhanced sensitivity also enables more precise and higher-cadence time-resolved observations. One important application of such observations is the characterization of transiting extrasolar planets, the science case that motivated the Nautilus concept described in this manuscript. As demonstrated, our baseline Nautilus concept may enable spectroscopic studies of approximately 1000 potentially habitable Earth-sized exoplanets. Such a survey would undoubtedly revolutionize astrophysics, planetary sciences, and astrobiology. The spectroscopic observations would enable the identification of several key atmospheric absorbers and would provide a pathway to determine or constrain the atmospheric composition. The survey envisioned here is distinct from other surveys proposed or planned owing to its very large sample size: the ability to study 1000 Earth- sized habitable zone planets may likely be essential for understanding the complexity and diversity of extrasolar planets (e.g., Seager 2014; Apai et al. 2017; Bean et al. 2017).

A sample size of 1000 planets would allow, for example, identification of potential trends between atmospheric absorbers and bulk properties of the planets. Comparison of the planets' atmospheric composition to the stellar irradiation received may allow empirical mapping of the inner and outer boundaries of the habitable zone (Kopparapu et al. 2013, 2018) and identification of possible atmospheric loss mechanisms (e.g., Owen \& Wu 2016). Seager (2014) argues that a sample size of 1000 or greater potentially Earth-like planets is likely required for a confident, statistical identification of life-bearing planets.

\subsection{Possible Pathway toward Large Diffractive Telescopes}

The fundamental theme of this paper is to explore the conjecture that large astronomical telescopes could be built based on multiorder diffractive engineered lenses and that such telescopes could be uniquely well suited to address astrophysical problems that require a large light-collecting area. The Nautilus observatory concept described herein is not a complete design reference mission, and it is not informed by detailed trade studies; instead, our study shows that possible solutions exist to most challenges MODE-lens-based telescope architectures may pose.

The technology readiness level of several components of the Nautilus concept is low; chief among these are the MODE lenses themselves with TRL2-3. In order to verify and realize the potential for very large MODE lenses for astronomical observations, a significant technology development and demonstration program is required. We briefly describe here a possible technology maturation pathway toward large MODE-lens-based telescopes.

First, technology development is required to demonstrate that high-quality MODE lenses can be fabricated and replicated with submeter diameters. Second, these lenses must be demonstrated in astronomical observations, firmly establishing such lenses at TRL3. Operational demonstration in thermalvacuum chambers will help move MODE lens technology to TRL5. A parallel technology development effort is required to scale up the fabrication/replication technology by building larger free-form optical fabrication and molding machines, preferably to $1-3 \mathrm{~m}$ diameters, and possibly beyond. With MODE lenses at TRL4-6, small-scale pathfinder, sciencedriven space missions will become viable, including small satellites and stratospheric balloons.

As MODE technology addresses a fundamental attribute of space telescopes (light collection), interesting science cases exist for even relatively small MODE-lens-based space telescopes. After a successful demonstration of the MODE lens technology in space or near-space environments on a SmallSat or stratospheric flight, a pair of $1-1.5 \mathrm{~m}$ diameter units may be flown as a NASA Small Explorer or Mid-Explorer mission, or a single $8 \mathrm{~m}$ diameter telescope could be flown as a Probe-class mission. If successful, a scaled-up and replicated version of these unit telescopes could serve as the first step in realizing the Nautilus observatory or an observatory with a similar scope. 
We note here that the initial steps of this process are underway: small (0.05 $\mathrm{m}$ diameter) MODE lenses have been fabricated, replicated, and demonstrated already; our team is currently working toward developing $0.24 \mathrm{~m}$ diameter MODElens-based telescopes, and their on-sky demonstration is scheduled for winter 2020. In addition, we are planning small satellite and balloon-borne MODE telescopes.

\section{Conclusions}

One of the most fundamental properties of astronomical telescopes is their light-gathering power; however, increases in mirror diameter over the past two centuries have been slow compared to performance increases seen in complementary fields (detectors, engineered materials, computer processors). In this study we described a very large astronomical telescope based on a novel, MODE lens design and an ultralight, inflatable spacecraft. Our concept focuses on the unique aspects of MODE-based telescopes, and it is not a complete, optimized mission concept. The notional Nautilus telescope concept introduced here is motivated by the science goal of surveying 1000 Earth-sized, potentially habitable exoplanets - a study that is important to understand the diversity of Earth-like planets but requires light-gathering power far beyond projected capabilities.

The key results of our study are as follows:

(1) Multiorder diffractive lenses provide ultralight and very large diameter alternatives to astronomical reflectors.

(2) MODE lenses potentially offer three key advantages over telescope mirrors: much lower weight per unit area, less sensitivity to misalignments/deformations, and efficient replicability through optical molding processes.

(3) We describe a novel and notional telescope concept that will allow transmission spectroscopy of 1000 transiting, Earthsized, potentially habitable planets at visual/near-infrared wavelengths.

(4) We evaluate four different target selection criteria for the exoplanet host stars (different spectral types) and assess the distances up to which a telescope must be capable of probing atmospheric biosignatures to search for life in 1000 Earth-sized habitable zone planets.

(5) We use the Planetary Spectrum Generator to calculate the expected transit spectra for some of the best-case and worstcase targets. We find that a $35 \times 8.5 \mathrm{~m}$ array of telescopes is sufficient to probe biosignatures in 1000 transiting Earth-sized habitable zone exoplanets.

(6) The Nautilus concept is based on a large array of identical unit telescopes, each equipped with a $2.5 \mathrm{~m}$ diameter lens optimized for wide-field imaging and exoplanet transit searches, and with an $8.5 \mathrm{~m}$ diameter MODE lens optimized for high-precision, moderate-resolution transit spectroscopy.

(7) Individual units can be used for wide-field surveys or targeted exoplanet transit searches, while the array-through the noncoherent combination of the light intensity signal from multiple units - enables the detection of faint light sources (e.g., very high redshift galaxies), as well as low-amplitude time-varying signal (e.g., exoplanet transit spectroscopy).

(8) The costs of the array are minimized by utilizing MODE lenses replicated through molding, by equipping each telescope with simple and identical instruments, and by launching 15 unit telescopes in a single launch.

(9) With two launches of next-generation rockets (SpaceX/ BFG or NASA SLS B2) enough unit telescopes can be launched for the Nautilus telescope array to provide a lightgathering power equivalent to a $50 \mathrm{~m}$ diameter space telescope.

(10) Although diffractive optical elements have flown as part of space instruments and small-scale MODE lenses exist, significant technology development is necessary before truly large-aperture MODE telescopes could be built. We discuss the key technology development challenges for MODE telescopes.

In summary, the concept described here offers a pathway to break away from the cost and risk growth curves defined currently by mirror technology and has the potential to enable very large and very lightweight, replicable technology for space telescopes. An example application of the Nautilus concept promises a revolutionary atmospheric biosignature survey of 1000 potentially Earth-like exoplanets.

The authors thank the anonymous referee, whose comments have improved the manuscript. We acknowledge helpful discussions and input from P. Apai, P. Atcheson, J. Breckinridge, B. Crill, J. M. Grunsfeld, B. T. Jannuzi, M. Marley, D. S. Lauretta, E. Mamajek, B. V. Rackham, N. Siegler, and $\mathrm{Z}$. Wang. The authors are particularly grateful for other members of the Nautilus team who contributed to the prototype MODE lens design, fabrication, and tests (O. Spires, Y.-S. Kim) and project management (C. Fellows). This study is funded in part by the Gordon and Betty Moore Foundation. We acknowledge A. Conti and the Northrop-Grumman Advanced Systems for a workshop on the future of exoplanet exploration and space telescopes, which seeded the current study. D.A. also acknowledges the NASA Exoplanet Exploration Program Office and the broader EXOPAG community for the development of the scientific and programmatic context that enabled this study. The results reported herein benefited from collaborations and/or information exchange within NASA's Nexus for Exoplanet System Science (NExSS) research coordination network sponsored by NASA's Science Mission Directorate.

Author contributions: D.A. initiated the Nautilus project, lead the mission concept definition and the development of the science case, created many of the figures, and drafted the manuscript. T.M. led the optical design and the invention of the MODE technology and contributed sections on the optical design. D.W.K. contributed sections on optical metrology and contributed to the conceptual design of the Nautilus system. A. B. led the transit simulations and the observation time assessment. G.S. contributed to the manuscript and to the definition of the science case. R.L. contributed to the optical fabrication section of the manuscript and leads optical fabrication of the MODE lens prototypes. J.A. contributed ideas to the Nautilus spacecraft concept and led the mission cost discussion.

Software: Python:NumPy/AstroPy.

\section{Appendix}

Table 7 summarizes the parameters for the calculation of the distance-dependence of the number of Earth-sized habitable zone transiting exoplanets described in Section 2.3. 
Table 7

Assumed Parameters for Sample Size Definition

\begin{tabular}{lclc}
\hline \hline Parameter & Value & Description & References \\
\hline$R_{*}(F)$ & 1.30 & Stellar radius for F7V-type star & Pecaut \& Mamajek (2013) \\
$R_{*}(G)$ & 0.95 & Stellar radius for G7V-type star & Pecaut \& Mamajek (2013) \\
$R_{*}(K)$ & 0.65 & Stellar radius for K7V-type star & Pecaut \& Mamajek (2013) \\
$R_{*}(M)$ & 0.12 & Stellar radius for M6.5V-type star & Pecaut \& Mamajek (2013) \\
\hline$M_{*}(F)$ & 1.21 & Stellar mass for F7V-type star & Pecaut \& Mamajek (2013) \\
$M_{*}(G)$ & 0.96 & Stellar mass for G7V-type star & Pecaut \& Mamajek (2013) \\
$M_{*}(K)$ & 0.65 & Stellar mass for K7V-type star & Pecaut \& Mamajek (2013) \\
$M_{*}(M)$ & 0.10 & Stellar mass for M6.5V-type star & Pecaut \& Mamajek (2013) \\
\hline$L_{*}(F)$ & 0.36 & Log. stellar luminosity for F7V-type star & Pecaut \& Mamajek (2013) \\
$L_{*}(G)$ & -0.12 & Log. stellar luminosity for G7V-type star & Pecaut \& Mamajek (2013) \\
$L_{*}(K)$ & -0.98 & Log. stellar luminosity for K7V-type star & Pecaut \& Mamajek (2013) \\
$L_{*}(M)$ & -3.09 & Log. stellar luminosity for M6.5V-type star & Pecaut \& Mamajek (2013) \\
\hline$\eta_{\oplus}(F)$ & 0.3 & Occurrence rate of hab. zone Earth-size planet, F7V-type host star & Based on SAG13 Meta-study \\
$\eta_{\oplus}(G)$ & 0.3 & Occurrence rate of hab. zone Earth-size planet, G7V-type host star & Based on SAG13 Meta-study \\
$\eta_{\oplus}(K)$ & 0.6 & Occurrence rate of hab. zone Earth-size planet, K7V-type host star & Based on SAG13 Meta-study \\
$\eta_{\oplus}(M)$ & 1.5 & Occurrence rate of hab. zone Earth-size planet, M6.5V-type host star & Based on SAG13 Meta-study \\
\hline$N_{10 \mathrm{pc}}(F)$ & 6 & Number of F stars within 10 pc & Henry et al. (2018) \\
$N_{10 \mathrm{pc}}(G)$ & 20 & Number of G stars within 10 pc & Henry et al. (2018) \\
$N_{10 \mathrm{pc}}(K)$ & 44 & Number of K stars within 10 pc & Henry et al. (2018) \\
$N_{10 \mathrm{pc}}(M)$ & 248 & Number of M stars within 10 pc & Henry et al. (2018) \\
\hline
\end{tabular}

Note.

${ }^{a}$ https://exoplanets.nasa.gov/exep/exopag/sag/

\section{ORCID iDs}

Dániel Apai (1) https://orcid.org/0000-0003-3714-5855

Alex Bixel (1) https://orcid.org/0000-0003-2831-1890

Glenn Schneider (1) https://orcid.org/0000-0002-4511-5966

Jonathan Arenberg (1) https://orcid.org/0000-0003-1096-5634

\section{References}

Apai, D., Ciesla, F., Mulders, G. D., et al. 2018, arXiv:1803.08682

Apai, D., Cowan, N., Kopparapu, R., et al. 2017, arXiv:1708.02821

Astropy Collaboration, Price-Whelan, A. M., \& Sipőcz, B. M. 2018, AJ, 156,123

Astropy Collaboration, Robitaille, T. P., \& Thomas, P. 2013, A\&A, 588, A33

Atcheson, P., Domber, J., Whiteaker, K., et al. 2014, Proc. SPIE, 9143, 91431W

Batalha, N. E., Lewis, N. K., Line, M. R., Valenti, J., \& Stevenson, K. 2018, ApJL, 856, L34

Bean, J. L., Abbot, D. S., \& Kempton, E. M.-R. 2017, ApJL, 841, L24

Bernet, S., \& Ritsch-Marte, M. 2017, OExpr, 25, 2469

Bixel, A., \& Apai, D. 2017, ApJL, 836, L31

Bixel, A., Rackham, B. V., Apai, D., et al. 2019, AJ, 157, 68

Borucki, W. J., Koch, D., Basri, G., et al. 2010, Sci, 327, 977

Cash, W., Kasdin, J., Seager, S., \& Arenberg, J. 2005, Proc. SPIE, 5899, 274

Catling, D. C., Krissansen-Totton, J., Kiang, N. Y., et al. 2018, AsBio, 18, 709

Checlair, J. H., Abbot, D. S., Webber, R. J., et al. 2019, arXiv:1903.05211

de Wit, J., Wakeford, H. R., Lewis, N. K., et al. 2018, NatAs, 2, 214

Faklis, D., \& Morris, G. M. 1995, ApOpt, 34, 2462

Fujii, Y., Angerhausen, D., Deitrick, R., et al. 2018, AsBio, 18, 739

Fulton, B. J., Petigura, E. A., Howard, A. W., et al. 2017, AJ, 154, 109

Gaudi, B. S., Seager, S., Mennesson, B., et al. 2018, arXiv:1809.09674

Gillon, M., Triaud, A. H. M. J., Demory, B.-O., et al. 2017, Natur, 542, 456

Gilmozzi, R., \& Spyromilio, J. 2007, Msngr, 127, 11

Grimm, S. L., Demory, B.-O., Gillon, M., et al. 2018, A\&A, 613, A68

Guyon, O., Hinz, P. M., Cady, E., Belikov, R., \& Martinache, F. 2014, ApJ, 780,171

Hansen, K. 2013, Developing Lightweight Optics for Space, https://str.llnl. gov/content/pages/january-2013/pdf/01.13.4.pdf

He, P., Li, L., Li, H., et al. 2014, Manufacturing Letters, 2, 17
Henry, T. J., Jao, W.-C., Winters, J. G., et al. 2018, AJ, 155, 265

Huang, C.-Y., Chen, C.-C., Chou, H.-Y., \& Chou, C.-P. 2013, OptRv, 20, 202 Huang, C.-Y., \& Liang, R. 2015, ApOpt, 54, 6979

Huang, R., Su, P., \& Burge, J. H. 2015, Proc. SPIE, 9575, 957515

Hvisc, A. M., \& Burge, J. H. 2007, Proc. SPIE, 6671, 66710A

Hyde, R. A. 1999, ApOpt, 38, 4198

Hyde, R. A., Dixit, S. N., Weisberg, A. H., \& Rushford, M. C. 2002, Proc. SPIE, 4849, 28

Johns, M., McCarthy, P., Raybould, K., et al. 2012, Proc. SPIE, 8444, 84441H Kiang, N. Y., Domagal-Goldman, S., Parenteau, M. N., et al. 2018, AsBio, 18,619

Kim, D. W., Oh, C.-j., Lowman, A., et al. 2016, Proc. SPIE, 9912, 99120F

Kite, E. S., \& Ford, E. B. 2018, ApJ, 864, 75

Kopparapu, R. K., Hébrard, E., Belikov, R., et al. 2018, ApJ, 856, 122

Kopparapu, R. K., Ramirez, R., Kasting, J. F., et al. 2013, ApJ, 765, 131

Kopparapu, R. K., Ramirez, R. M., SchottelKotte, J., et al. 2014, ApJL, 787, L29

Kreidberg, L., Bean, J. L., Désert, J.-M., et al. 2014, Natur, 505, 69

Lee, W. B., \& Cheung, B. C. F. 2003, Surface Generation in Ultra-precision Diamong Turning: Modeling and Practices (1st ed.; New York: Wiley)

Lo, A. S., \& Arenberg, J. 2006, Proc. SPIE, 6265, 626522

Martin, S., Rud, M., Mawet, D., et al. 2018, Proc. SPIE, 10698, 106980T

Meadows, V. S., Reinhard, C. T., Arney, G. N., et al. 2018, AsBio, 18, 630

Milster, T. D. 2018, OPTI 505R: Diffraction and Interferometry, Lecture Notes, Chapter 5.3 (Tucson, AZ: Univ. Arizona Press)

Milster, T. D., Apai, D., \& Johnson, L. C. 2018a, A Multiorder Diffractive Fresnel Lens (MOD-DFL) and Method for Enhancing Images That Are Captured Using the MOD-DFL, U.S. Provisional Pat. Ser. No. 62/695,531

Milster, T. D., Apai, D., \& Johnson, L. C. 2018b, A Multiorder Diffractive Fresnel Lens (MOD-DFL) and Systems That Incorporate the MOD-DFL, U.S. Provisional Pat. Ser. No. 62/691,892

Nakai, T. 2003, Diffractive Optical Element, U.S. Patent No. 6,587,272

Nelson, J., Scordato, M., Schwertz, K., \& Bagwell, J. 2015, Proc. SPIE, 9633 , 96331L

Oh, C. J., Lowman, A. E., Smith, G. A., et al. 2016, Proc. SPIE, 9912, 991200

Owen, J. E., \& Wu, Y. 2016, ApJ, 817, 107

Parks, R. E. 2008, Proc. SPIE, 7071, 70710B

Parks, R. E. 2010, Proc. SPIE, 7793, 779304

Parks, R. E. 2011, Proc. SPIE, 8126, 81260D

Pecaut, M. J., \& Mamajek, E. E. 2013, ApJS, 208, 9

Rackham, B. V., Apai, D., \& Giampapa, M. S. 2018, ApJ, 853, 122 
Rackham, B. V., Apai, D., \& Giampapa, M. S. 2019, AJ, 157, 96

Ramirez, R. M., Abbot, D. S., Fujii, Y., et al. 2019, arXiv:1903.03706

Ramos-Izquierdo, L., Scott, V. S., III, Connelly, J., et al. 2009, ApOpt, 48, 3035

Rauer, H., Catala, C., Aerts, C., et al. 2014, ExA, 38, 249

Ricker, G. R., Winn, J. N., Vanderspek, R., et al. 2015, JATIS, 1, 014003

Rogers, L. A. 2015, ApJ, 801, 41

Sanders, G. H. 2013, JApA, 34, 81

Seager, S. 2014, PNAS, 111, 12634

Seager, S., Bains, W., \& Petkowski, J. J. 2016, AsBio, 16, 465

Seager, S., \& Deming, D. 2010, ARA\&A, 48, 631

Siegler, N. 2018, AAS Meeting Abstracts, 231, 104.03

Staasmeyer, J.-H., Wang, Y., Liu, G., Dambon, O., \& Klocke, F. 2016, Proc. SPIE, 9949, 994904

Stone, T., \& George, N. 1988, ApOpt, 27, 2960
Su, P., Parks, R. E., Wang, L., Angel, R. P., \& Burge, J. H. 2010, ApOpt, 49,4404

Su, P., Wang, S., Khreishi, M., et al. 2012, Proc. SPIE, 8450, 84500W

Sweatt, W. C. 1977, JOSA, 67, 803

The LUVOIR Team 2018, arXiv:1809.09668

The OST Mission Concept Study Team 2018, arXiv:1809.09702

Trauger, J. T., \& Traub, W. A. 2007, Natur, 446, 771

Trumper, I., Jannuzi, B. T., \& Kim, D. W. 2018, OptLE, 104, 22

van der Walt, S., Colbert, S. C., \& Varoquaux, G. 2011, CSE, 13, 22

Villanueva, G. L., Smith, M. D., Protopapa, S., Faggi, S., \& Mandell, A. M. 2018, JQSRT, 217, 86

Wertz, J. R., Everett, D. F., \& Puschell, J. J. 2011, Space Mission Engineering: The New SMAD (3rd ed.; Cleveland, OH: Microcosm Press)

Zhang, L., \& Liu, W. 2017, FrME, 12, 3

Zhang, Z., Zhou, Y., Rackham, B. V., \& Apai, D. 2018, AJ, 156, 178 